Pacific Journal of Mathematic 


\section{APPLICATIONS OF THE RAYLEIGH RITZ METHOD TO VARIATIONAL PROBLEMS}

\section{J. INDRITZ}

Introduction. Let $R$ be a bounded either simply or multiply connected plane region with boundary $\Gamma$, consisting of a finite number of non-intersecting simply closed regular arcs of class $c^{k}$. A plane curve is a regular are if the defining functions $x(t), y(t), a \leq t \leq b$ have continuous derivatives with $x^{\prime}(t)^{2}+y^{\prime}(t)^{2} \Longrightarrow 0$ on $a \leq t \leq b$. A regular arc is of class $c^{k}$ if the defining functions $x(s), y(s), s$ being arc length, have continuous derivatives of order $k$. We shall say a function $h(x, y)$ defined on $\bar{R}=R+\Gamma$ is of class $c^{k}$ if the partial derivatives of $h$ of order $r, 0 \leq r \leq k$ exist in $R$ and have limits on $I$ so as to define functions continuous on $\bar{R}$. Let $g(x, y)$ be a given function of class $c^{k}$ on $\bar{R}$. The main problem considered is that of finding the function $\psi_{0}$ which yields minimum value to the functional

$$
I[\psi]=\iint_{R}\left(a \psi_{x}^{2}+b \psi_{y}^{2}+c \psi^{2}+2 f \psi\right) d x d y
$$

defined over the admissible class of functions $\psi$ which are of class $c^{k}$ on $\bar{R}$ and assume the values of $g$ on $\Gamma$.

We shall assume $a(x, y)>0, b(x, y)>0, c(x, y) \geq 0$ on $\bar{R} ; a, b, c$ bounded and integrable in $\bar{R} ; f(x, y)$ integrable in $\bar{R}$. In the sequel, unless otherwise specified, integrations will be taken over $R$ and the symbol $R$ omitted.

Let $G(x, y)$ be of class $c^{k}$ on $\bar{R}$, vanishing on $\Gamma$, positive in $R$, with normal derivative $\partial G / \partial \nu$ on $\Gamma$ different from 0 . We show that, if $k \geq 3$, every admissible function $\psi$ has a uniformly convergent expansion on $\bar{R}$

$$
\psi=g+\sum_{i=1}^{\infty} b_{i} f_{i}(x, y)
$$

where $f_{i}$ are obtained by a Gram-Schmidt process from the functions $\left\{G x^{i} y^{j}\right\} i, j=0,1,2, \cdots$ and $b_{i}$ are generalized Fourier coefficients connected with the quadratic functional

$$
D[\psi]=\iint\left(a \psi_{x}^{2}+b \psi_{y}^{2}+c \psi^{2}\right) d x d y .
$$

Received February 6, 1954. Presented to the American Mathematical Society August 1953. The preparation of this paper was sponsored, in part, by the Office of Naval Research, Contract $\mathrm{N}$ onr-386(00). It is a part of the author's thesis under the helpful direction of Professor S.E. Warschawski. 
In fact, $b_{i}=D\left[\psi-g, f_{i}\right]$ where

$$
D[\xi, \eta]=\iint\left(a \xi_{x} \eta_{x}+b \xi_{y} \eta_{y}+c \xi \eta\right) d x d y .
$$

An estimate of the error obtained by using for $\psi$ only the first $n$ terms of the expansion is given in terms of $n$ and $k$. Sufficient conditions are obtained for the convergence of

$$
\nabla\left[g+\sum_{i=1}^{n} b_{i} f_{i}\right]
$$

to $\nabla \psi$ and an estimate is given for the rate of convergence.

In particular, if $\psi_{0}$ is an admissible function minimizing $I[\psi]$, then the expansion

$$
\psi_{0}=g+\sum_{i=1}^{\infty} a_{i} f_{i}
$$

yields an explicit solution for $\psi_{0}$, since the coefficients $a_{i}$ are given, in this case, by

$$
a_{i}=-\iint f f_{i} d x d y-D\left[g, f_{i}\right]
$$

which are independent of $\psi_{0}$.

The problem of minimizing the functional $I[\psi]$, with $g \equiv 0$, has been studied by Kryloff and Bogoliubov [4] and by Kantorovitch [2], both obtaining estimates for convergence to $\psi_{0}$ of functions obtainable by the Rayleigh Ritz method. The first paper deals with convex regions $R$, the second with regions $R$ bounded by $x=0, x=1, y=g(x)$, $y=h(x) ; h>g$ on $0 \leq x \leq 1$. Neither obtains an explicit solution for $\psi_{0}$ nor studies the convergence of the derivatives.

In the final section of this paper, we assume the existence of a function $\psi_{0}$ yielding minimum value, for $p \geq 1$, to

$$
D^{p}[\psi]=\iint_{R}\left(a \psi_{x}^{2}+b \psi_{y}^{2}+c \psi^{2}\right)^{p} d x d y, \quad \psi=g \quad \text { on } \quad \Gamma
$$

and obtain an estimate for the rate of convergence to $\psi_{0}$ of functions obtained by the Rayleigh Ritz method.

$\S 1$. Preliminary Considerations. A variation $v$ shall mean a function of class $c^{k}$ on $\bar{R}$ vanishing on $\Gamma$. Form the Hilbert space $H$ by completing the linear manifold $V$ of variations $v$ using the positive definite quadratic form $D[v]$ as the square of the norm of a variation. If $h \in H$, we represent the norm of $h$ by $h$. If $\xi$ and $\eta$ are variations, the inner product will be

$$
(\xi, \eta)=D[\xi, \eta] \text {. }
$$


Let $f_{i}$ be any complete orthonormal set of variations in $H$. If $\psi^{\prime}$ is admissible, then $\psi-g$ is a variation and thus expressible in $H$ as

$$
\psi-g=\sum_{i=1}^{\infty} b_{i} f_{i}
$$

with $b_{i}=D\left[\psi-g, f_{i}\right]$.

If $\psi_{0}$ is an admissible function yielding a minimum value to $I[\psi]$, if $\lambda$ is real, and $v$ is a variation, then $\psi_{0}+\lambda v$ is admissible, and

$$
I\left[\psi_{0}\right] \leq I\left[\psi_{0}+\lambda v\right]=I\left[\psi_{0}\right]+\lambda\left(2 D\left[\psi_{0}, v\right]+\iint 2 f v d x d y\right)+\lambda^{2} D[v] .
$$

This implies that the coefficient of $\lambda$ must vanish so that

$$
D\left[\psi_{0}, v\right]=-\iint f v d x d y
$$

and

$$
I\left[\psi_{0}+\lambda v\right]=I\left[\psi_{0}\right]+\lambda^{2} D[v]
$$

for every variation $v$.

The first relation shows that the Fourier coefficients of $\psi_{0}-g$,

$$
a_{i}=D\left[\psi_{0}-g, f_{i}\right]=D\left[\psi_{0}, f_{i}\right]-D\left[g, f_{i}\right]=-\iint f f_{i} d x d y-D\left[g, f_{i}\right]
$$

are independent of $\psi_{0}$.

The second relation implies that if $\psi$ is admissible,

$$
I[\psi]=I\left[\psi_{0}+\psi-\psi_{0}\right]=I\left[\psi_{0}\right]+D\left[\psi-\psi_{0}\right] .
$$

Thus if

$$
\phi_{n}=g+\sum_{i=1}^{n} a_{i} f_{i}
$$

then

$$
0=\lim _{n \rightarrow \infty}\left\|\psi_{0}-\left(g+\sum_{i=1}^{n} a_{i} f_{i}\right)\right\|^{2}=\lim _{n \rightarrow \infty} D\left[\psi_{0}-\phi_{n}\right]=\lim _{n \rightarrow \infty} I\left[\phi_{n}\right]-I\left[\psi_{0}\right]
$$

so that $\phi_{n}$ is a minimizing sequence.

Moreover,

$$
D\left[\psi_{0}-g-\sum_{i=1}^{n} c_{i} f_{i}\right]
$$

is a minimum when $c_{i}=a_{i}$ implying that $\phi_{n}$ are chosen to yield minimum value to $I\left[\psi_{n}\right]-I\left[\psi_{0}\right]$ and hence to $I\left[\psi_{n}\right]$ in the class of functions

$$
\psi_{n}=g+\sum_{i=1}^{n} c_{i} f_{i}
$$

Thus $\phi_{n}$ may be obtained by the Rayleigh Ritz process applied to the functional $I[\psi]$. 
We will prove, in Theorem 1, that the class of functions $\{G P\}$ where $P$ is a polynomial in $x$ and $y$, is dense in $H$. This class is the linear manifold determined by the set $\left\{G x^{i} y^{p}\right\}$, a set linearly independent in $H$. For, if

$$
v==\sum_{i=1}^{m} \sum_{i=1}^{n} \alpha_{i j} G x^{i} y^{j}
$$

then $D[v]=0$ implies $\alpha_{i j}=0$.

It follows that we can obtain an orthonormal set $f_{i}$ complete in $H$ by orthonormalizing the set $\left\{G x^{i} y^{j}\right\}$. Let

$$
\begin{aligned}
& v_{1}=G x^{0} y^{0} \\
& v_{2}=G x^{1} y^{0}, \quad v_{3}=G x^{0} y^{1} \\
& \vdots \\
& v_{\substack{k(k+1)+1 \\
2}}=G x^{k} y^{0}, \cdots, v_{k(k+1)+k+1}^{2}=G x^{0} y^{k} .
\end{aligned}
$$

Then

$$
\begin{aligned}
& f_{n}=\frac{v_{n}-\sum_{j=1}^{n-1} f_{j}\left(v_{n}, f_{j}\right)}{\left\|v_{n}-\sum_{j=1}^{n-1} f_{j}\left(v_{n}, f_{j}\right)\right\|}
\end{aligned}
$$

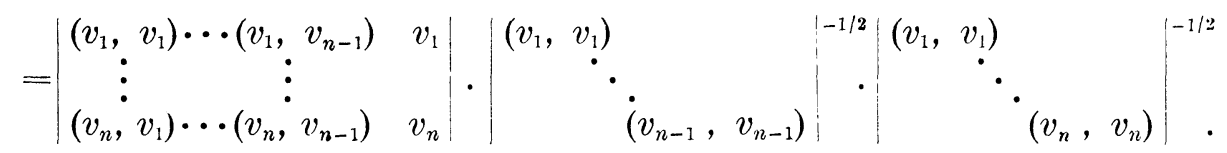

The function $f_{n}$ is of the form $G P_{n}$, where the degree of the polynomial $P_{n}$ is that of $v_{n} / G$. If $v_{n}=G x^{r} y^{s}$ with $r+s=k$, then

$$
\frac{k(k+1)}{2}+1 \leq n \leq \frac{k(k+1)}{2}+k+1
$$

so that $k^{2}<k(k+1)<2 n-2$ and the degree $k$ of $P_{n}$ is less than $\sqrt{ } 2 n-2$. Similarly $k$ is greater than $\sqrt{ } 2 n-2$.

§2. The Minimizing sequence. We shall use certain approximation theorems which can be derived by methods used by Mickelson [5] To simplify the notation, let

$$
\begin{gathered}
x=\left(x_{1}, \cdots x_{s}\right), \\
x^{(1)}=\left(x_{1}^{(1)}, \cdots x_{s}^{(1)}\right),
\end{gathered}
$$

${ }^{1}$ For detailed proofs of Lemmas 1, 2 see J. Indritz "Applications of the Rayleigh Ritz method to the solutions of partial differential equations" $\mathrm{Ph}$. D. Thesis, U. of Minnesota, 1953. 


$$
\begin{aligned}
& f(x)=f\left(x_{1}, \cdots, x_{s}\right), \\
& f_{r}(x)=\frac{\partial^{r_{1}+\cdots+r_{s}}}{\partial x_{1}^{{ }^{r_{1}} \cdots \partial x_{s}^{r} s}}\left[f\left(x_{1}, \cdots, x_{s}\right)\right], \\
& \left\|x^{(1)}-x^{(2)}\right\|=\sqrt{\sum_{i=1}^{s}\left(x_{i}^{(1)}-x_{i}^{(2)}\right)^{2} .}
\end{aligned}
$$

The modulus of continuity for a function $f$ defined over a closed set $A: \quad-1 \leq x_{i} \leq 1(i=1, \cdots, s)$ is

$$
\Omega(\delta, f)=\sup \left|f\left(x^{(1)}\right)-f\left(x^{(2)}\right)\right|
$$

for all points $x^{(1)}, x^{(2)}$ in $A$ with $\left\|x^{(1)}-x^{(2)}\right\| \leq \delta$. The uniform modulus of continuity of a finite number of functions $f_{1}, \cdots, f_{N}$ is the largest of the moduli of each $f_{i}$ for each $\delta$.

Lemma 1. Let $F(\theta)$ be a continuous periodic function of period $2 \pi$ in each $\theta_{i}$ and of class $c^{k}$. Let $\omega(\delta)$ be the uniform modulus of continuity of the partial derivatives of $F$ of order 1 to $k$ for $\delta \leq \pi / \mathrm{s}$. Let $j \leq k$. Then, corresponding to every set $m_{1}, \cdots, m_{s}$ of positive integers, there is a trigonometric sum $T^{m}$ of order at most $m_{i}$ in $\theta_{i}$ such that

$$
\left|F_{r}(\theta)-T_{r}^{m}(\theta)\right| \leq K_{1}\left(\sum_{i=1}^{s} \frac{1}{m_{i}}\right)^{k-j} \sum_{i=1}^{s} \omega\left(\frac{1}{m_{i}}\right) \text { for } \quad 0 \leq r_{1}+\cdots+r_{s} \leq j
$$

where $K_{1}$ is a constant independent of $F, s, m_{i}$.

If the partial derivatives of order 1 to $k$ satisfy

$$
\left|F_{r}\left(\theta^{(1)}\right)-F_{r}\left(\theta^{(2)}\right)\right| \leq L\left(\sum_{i=1}^{s}\left|\theta_{i}^{(1)}-\theta_{i}^{(2)}\right|\right)
$$

then

$$
\left|F_{r}(\theta)-T_{r}^{m}\right| \leq L K_{2}\left(\sum_{i=1}^{s} \frac{1}{m_{i}}\right)^{k-j+1} \quad 0 \leq r_{1}+\cdots+r_{s} \leq j
$$

where $K_{2}$ is also a constant independent of $F, s, m_{i}$.

If $F$ is even in each $\theta_{i}$ separately, $T$ contains only cosine terms.

Lemma 2. Let $f(x)$ be of class $c^{k}$ in the set $A:-1 \leq x_{i} \leq 1(i=1, \cdots, \mathrm{s})$. Let $M$ be the maximum of the absolute values of the derivatives of order. 1 to $k$, and $\Omega(\delta)$ the uniform modulus of continuity of the derivatives of order $k$. Let $B$ denote a closed set interior to $A$. Let $j \leq k$. Then, for every set of positive integers $m_{1}, \cdots, m_{s}$ with $m_{i} \geq k$ there is a polynomial $P^{m}$ of order at most $m_{i}$ in $x_{i}$ such that 


$$
\left|f_{r}(x)-P_{r}^{m}(x)\right| \leq K_{3}\left(\sum_{i=1}^{s} \frac{1}{m_{i}}\right)^{k-j} \sum_{i=1}^{s} \Omega\left(\begin{array}{c}
1 \\
m_{i}
\end{array}\right)
$$

for $x$ in $B$ and $0 \leq r_{1}+\cdots+r_{s} \leq j$. Here $K_{3}$ is a constant independent of $f$ and $m_{i}$.

If also, the k-th partial derivatives of $f(x)$ satisfy a Lipschitz condition with parameter $\lambda$, then, for $x$ in $B$,

$$
\left|f_{r}(x)-P_{r}^{m}(x)\right| \leq K_{4}\left(\sum_{i=1}^{s} \frac{1}{m_{i}}\right)^{k-j+1} \quad \text { for } \quad 0 \leq r_{1}+\cdots+r_{s} \leq j,
$$

and where $K_{4}$ is a constant independent of $f$ and $m_{i}$.

To apply the lemmas to a function defined over the region $R$, we shall extend the domain of definition of the function. The question arises whether the differentiability properties of the function are maintained under the extension. The answer depends upon the properties of the boundary $\Gamma$ of $R$. For example, Hirschfeld [1] has shown that even a cusp in the complementary region may prevent $c^{1}$ extension of a function of class $c^{\infty}$ on a closed set through a continuous boundary arc. Whitney [6] has given a different definition for a function to be of class $c^{k}$ in a closed set $A$. If $f$ is of Whitney class $c^{k}$ in $A$, then there exists an extension $F$ to the whole plane $E_{2}$ which is of class $c^{k}$ in the ordinary sense on $E_{2}$ and is analytic in $E_{2}-A$. The derivatives of $F$ of order $\leq k$ coincide with those of $f$ at any interior point of $A$. Moreover Whitney [7] has shown the following: Let (a) $f$ be of class $c^{k}$ on $R+\Gamma$, where $R$ is a region, $\Gamma$ its boundary, in the sense we have defined in the introduction, and (b) $R$ have the property " $P$ ", that any two points $P_{1}, P_{2}$ in $R$, whose linear distance apart may be represented by $P_{1}-P_{2}$, can be joined by a rectifiable curve in $R$ of length $L$, with $L / \| P_{1}-P_{2}$ bounded uniformly with respect to $P_{1}$ and $P_{2}$; then $f$ is also of Whitney class $c^{k}$ and thus can be extended to $E_{2}$ to be of class $c^{k}$ on $E_{2}$.

For our purposes we assume $R$ to be a bounded region with boundary $\Gamma$ consisting of a finite number of non-intersecting simply closed regular $\operatorname{arcs} \Gamma_{i}$ and we will show $R$ has property " $P$ ".

Choose, for each $\Gamma_{i}$, a $\delta>0$ such that no two tangents to $\Gamma_{i}$ on any portion of arc length $<\delta$ make with each other an angle greater than $5^{\circ}$. We may choose $\delta$ independent of $i$ and smaller than onefourth the distance between any two $\Gamma_{i}$. Now fix $i$, and let $P_{1}, P_{z}$ be points on $\Gamma_{i}$ on a subarc of length $<\delta$. There is a point $Q$ on that subarc between $P_{1}$ and $P_{2}$ such that the tangent line at $Q$ is parallel to the chord $P_{1} P_{2}$. Set up an $(x, y)$ coordinate system at $Q$, using the tangent line as $x$-axis, the normal as $y$-axis, and note that the subarc 
considered has an equation $y=y(x)$ of class $c^{1}$ in view of the implicit function theorems. Let $P_{1}=\left(x_{1}, y_{1}\right), P_{2}=\left(x_{2}, y_{2}\right),\left\|P_{1}-P_{2}\right\|=$ distance between $P_{1}$ and $P_{2},\left\|\overparen{P_{1} P_{2}}\right\|=$ length of the subarc joining $P_{1}$ to $P_{2}$. Then $\| P_{1}-P_{2}=\left|x_{1}-x_{2}\right|$ and $\left|y^{\prime}(x)\right| \leq 1$ so that

$$
\begin{aligned}
& \left\|P_{1}-P_{2}\right\| \leq \| \overparen{P}_{1} P_{2}|=| \int_{x_{1}}^{x_{2}} \sqrt{ } 1+y^{\prime 2} d x \mid \\
& \leq \sqrt{2}\left|x_{1}-x_{2}\right|=\sqrt{2}\left\|P_{1}-P_{2}\right\| .
\end{aligned}
$$

Moreover, since $\tan 5^{\circ}<1 / 10$, the mean value theorem shows that $\sup |y(x)| \leq\left\|P_{1}-P_{2}\right\| / 10$.

We shall also use the well known property that if $\Gamma_{i}$ is a regular arc, there is an $\omega_{1}>0$ such that for any subarc joining points $P_{3}, P_{4}$ on $\Gamma_{i}$, we have $\left\|\overparen{P}_{3} P_{4}\right\| /\left\|P_{3}-P_{4}\right\| \leq \omega_{1}$. $\omega_{1}$ can be chosen independent of $i$.

Now suppose $S_{1}, S_{2}$ are any two points interior to the region $R$. If the segment $S_{1} S_{2}$ is interior to $R$, we of course have $\| S_{1} S_{2} \mid$ $\left\|S_{1}-S_{2}\right\|=1$ by using the segment as the arc. Otherwise, let $Q_{1}$ be the first intersection of the directed line $S_{1} S_{2}$ with the boundary, say with $\Gamma_{1}$. Let $Q_{1}^{1}$ be a point on $S_{1} Q_{1}$ in $R$. Let $Q_{2}$ be the first point of intersection of the directed line $S_{2} S_{1}$ with $\Gamma_{1}$ and $Q_{2}^{1}$ a point in $R$ on $S_{2} Q_{2}$ such that the open interval $Q_{2} Q_{2}^{1}$ is also in $R$. Note that $Q_{1}$ and $Q_{2}$ may coincide. If $Q_{2}^{1} S_{2}$ is not in $R$, let $Q_{3}$ be the first point of intersection of the directed line $Q_{2}^{1} S_{2}$ with the boundary, say with $\Gamma_{2}$ and $Q_{3}^{1}$ in $R$ and on $Q_{2}^{1} Q_{3}$. Let $Q_{t}$ be the first point of intersection of the directed line $S_{2} Q_{2}^{1}$ with $\Gamma_{2}$ and $Q_{4}^{1}$ a point in $R$, on $Q_{4} S_{2}$, with the open interval $Q_{4} Q_{4}^{1}$ in $R$. Continuing in this way, after at most $n$ steps, we form a finite sequence of points $Q_{0}^{1}=S_{1}, Q_{1}^{1}, Q_{2}^{1}, \cdots, Q_{2 m}^{1}, Q_{2 m+1}^{1}$ $=S_{2}$ such that $Q_{2 k-1}$ and $Q_{2 k}$ are on the same regular arc, and the lines joining $Q_{2 k}^{1}$ to $Q_{2 k+1}^{1}, k=0, \cdots, m$ are in $R$. If we can show there is an $\omega>0$, independent of the points, and $\operatorname{arcs} \lambda_{1}$ in $R$ joining consecutive points $Q_{j}^{1}$ to $Q_{j+1}^{1}$ such that $\left\|\overparen{Q_{j}^{1} Q_{j+1}^{1}}\right\| \leq \omega\left\|Q_{j}^{1}-Q_{j+1}^{1}\right\|$, then we can attain the desired results by addition. It suffices to show that $Q_{1}^{1}$ and $Q_{2}^{1}$ and an arc $\lambda$ joining $Q_{1}^{1}$ to $Q_{2}^{1}$ and in $R$ may be chosen so that $\| \overparen{Q_{1}^{1} Q_{2}^{1}}$ $\leq \omega\left\|Q_{1}^{1}-Q_{2}^{1}\right\|$. Suppose first that $Q_{1}$ and $Q_{2}$ coincide. A sufficiently small circle with $Q_{1}$ as center will have one of the arcs cut off by $S_{1} S_{2}$ entirely in $R$ and we may choose $Q_{1}^{1}$ and $Q_{2}^{1}$ as the intersections of $S_{1} S_{2}$ with this circle. In this case

$$
\left\|\overparen{Q_{1}^{1} Q_{2}^{1}}\right\|=\frac{\pi}{2}\left\|Q_{1}^{1}-Q_{2}^{1}\right\|
$$

Otherwise, let $L$ be the length of an arc on $\Gamma_{1}$ joining $Q_{1}$ to $Q_{2}$. 
Divide this arc into $N$ equal segments of length $\beta=L / N$ where $N$ is sufficiently large so that $\beta<\delta$. Draw circles of radius $r=\beta / \sqrt{ } 2$ about each of the division points and the end points. We first show that consecutive circles intersect. If $R_{1}$ and $R_{2}$ are two consecutive centers, (1) implies

$$
\left\|R_{1}-R_{2}\right\| \leq \beta \leq V 2\left\|R_{1}-R_{2}\right\|
$$

so that

$$
\underset{2}{\| R_{1}-R_{2}}\left\|\leq \frac{\| R_{1}-R_{2}}{\sqrt{2}}\right\|_{\leq} \leq \frac{\beta}{\sqrt{2}} \leq\left\|R_{1}-R_{2}\right\|,
$$

and the circles must intersect.

Moreover, since $r \geq\left\|R_{1}-R_{2}\right\| / V 2$, the semi-length $\tau$ of the common chord is

$$
\tau=\sqrt{ } r^{2}-\frac{\left\|R_{1}-R_{2}\right\|^{2}}{4} \geq \frac{\left\|R_{1}-R_{2}\right\|}{2}
$$

whereas the arc joining $R_{1}$ to $R_{2}$, has distance $\left\langle\left\|R_{1}-R_{2}\right\| / 10\right.$ from the chord. Hence the arc lies entirely within the circles.

Now let $Q_{1}^{1}$ be an intersection of $S_{1} S_{2}$ with the circle whose center is $Q_{1}$ and $Q_{2}^{1}$ an intersection of $S_{1} S_{2}$ with the circle whose center is $Q_{2}$, the points being chosen to lie in $R$ and have the desired properties. Starting from $Q_{1}^{1}$ we may proceed to $Q_{2}^{1}$ via the circumferences of the circles. The total length of the curve thus formed will be less than

$$
(N+1) 2 \pi \underset{V}{\beta}=\frac{N+1 \quad 2 \pi}{N \quad V 2} L \leq \frac{4 \pi}{V} L
$$

and

$$
\frac{\left\|Q_{1}^{1} Q_{2}^{1}\right\|}{\left\|Q_{1}^{1}-Q_{2}^{1}\right\|} \leq \frac{4 \pi}{V 2} \frac{L}{\left\|Q_{1}^{1}-Q_{2}^{1}\right\|} \leq \frac{4 \pi}{V 2} \frac{L}{\left\|Q_{1}-Q_{2}\right\|} \leq \frac{4 \pi}{V} \omega_{1}
$$

This concludes the proof that $R$ has property " $P$ "'.

We will be particularly interested in extending a function of the form $v(x, y) / G(x, y)$ where $G(x, y)>0$ in $R, \partial G / \partial \nu>0$ on $\Gamma, G=v=0$ on $\Gamma$ and we seek differentiability conditions on $v$ and $G$ which insure that $v / G$ is of class $c^{k}$ on $R+I$. Here again the nature of the boundary is of importance. The next two lemmas deal with this problem. The letter $P$ will refer to a point in $R$ and $Q$ to a point on $I^{\prime}$, the boundary of $R$. By a neighborhood $N(Q)$ in $R+\Gamma$ we will mean a set of points $S$ in $R+\Gamma$ such that for some sufficiently small circle with center at $Q$, every point of the circle which lies in $R+\Gamma$ also lies in $S$. 
Lemma 3. a) Let $R$ be a region bounded by $\Gamma^{\prime}$, a finite number of closed Jordan curves, no two having a point in common. Let $r$ be a regular subarc of $\Gamma$, and $Q_{0}$ an interior point of $\gamma$. Let $N$ be the normal to $\gamma$ at $Q_{0}$. Then there is a neighborhood $N\left(Q_{0}\right)$ in $R+\Gamma$ such that through each point $P$ in $R N\left(Q_{0}\right)$, the line parallel to $N$ cuts $\gamma_{0}=\gamma N\left(Q_{0}\right)$ in one and only one point $Q, P Q$ lies in $N\left(Q_{0}\right)$, and $Q$ ranges over $\gamma_{0}$.

b) Let $\psi(x, y)$ be of class $c^{1}$ in $R N\left(Q_{0}\right)$ and suppose $\psi, \psi_{x}$, $\psi_{y}$ have continuous limits on $\gamma_{0}$. Define $(\partial \psi / \partial s)(P)$ to be the derivative at $P \in$ $R N\left(Q_{0}\right)$ in the direction of the tangent at the corresponding point $Q$ on $\gamma_{0}$. The derivative $(\partial \psi / \partial s)(P)$ has continuous limits on $\gamma_{0}$ which we will denote by $(\partial \psi / \partial s)(Q)$.

If $\psi=0$ on $\gamma_{0}$, then $(\partial \psi / \partial s)(Q)=0$ for $Q$ on $\gamma_{0}$.

Proof. Let $\gamma$ be given by $x(t), y(t)$ and $Q_{0}$ defined by the parameter value $t_{0}$. Let $(\xi, \eta)$ be rectangular axes along the tangent and normal at $Q_{0}$. In a suitable neighborhood of $t_{0}, t_{1}<t<t_{2}$, defining an arc $\lambda_{0}$ containing $Q_{0}, \gamma$ admits a representation $\gamma=\gamma(\xi)$. We may assume $\lambda_{0}$ so small that no two tangents to it make with each other an angle greater than $5^{\circ}$. There is a positive distance $d$ between $\Gamma-\lambda_{0}$ and the arc $\lambda_{1}$ defined by the parameter range $\left(t_{1}+t_{0}\right) / 2<t<\left(t_{0}+t_{2}\right) / 2$. Take

$$
\delta<\min \left[d,\left|\xi\left(t_{0}\right)-\xi\left(\left(t_{0}+t_{2}\right) / 2\right)\right|,\left|\xi\left(t_{0}\right)-\xi\left(\left(t_{0}+t_{1}\right) / 2\right)\right|\right]
$$

and draw a square $T$ of side $\delta$ with sides parallel to the $(\xi, \eta)$ axes and center at $Q_{0}$. Let $\gamma_{0}=\gamma T$, the projection of $R T$ on $\gamma$ by lines parallel to $N$, and let $\gamma_{l}$ be the arcs formed by displacing $\gamma_{0}$ a distance $h$ parallel to itself into $R$ along $N$. For $h<h_{1}$ sufficiently small, $\gamma_{h} \subset T$. The regular are $\gamma_{0}$ may be given a representation $x=x(s), y=y(s)$, $0 \leq s \leq L$, in terms of arc length $s$, where $L$ is the length of $\gamma_{0}$. Then $\gamma_{h}$ is given by

$$
x=x(s)+h \cos \alpha, \quad y=y(s)+h \cos \beta,
$$

where $\cos \alpha, \cos \beta$ are the direction cosines of the line $N$ directed inward into $R$. The neighborhood $N\left(Q_{0}\right)$ may be chosen as given by these equations with $0<s<L .0 \leq h<h_{1}$.

It is clear that

$$
\frac{\partial \psi}{\partial s}=\frac{\partial \psi}{\partial x} \cdot \frac{d x}{d s}+\frac{\partial \psi d y}{\partial y} \frac{d y}{d s}
$$

has continuous limits on $\gamma_{0}$. Write

$$
\partial{ }^{\partial}(P)=\frac{\partial \psi}{\partial s}(x(s)+h \cos \alpha, y(s)+h \cos \beta)=F(s, h) .
$$


If $\lambda$ is any closed subarc of $\gamma_{0}$, we have

$$
\lim _{h \rightarrow 0} F(s, h)=\frac{\partial \psi}{\partial s}(Q)
$$

uniformly in $s$.

Along $\gamma_{h}$ we have

$$
\psi\left(P_{2}\right)-\psi\left(P_{1}\right)=\int_{s_{1}}^{s_{2}} F(s, h) d s
$$

where $P_{1}$ and $P_{2}$ are points on $\gamma_{h}$ corresponding to points $Q_{1}$ and $Q_{2}$ on $\lambda$ with parameter values $s_{1}$ and $s_{2}$. As $h$ approaches 0 , the limits on the integral remain fixed. Since $\psi=0$ on $\lambda$, we find, by letting $h \rightarrow 0$,

$$
0=\int_{s_{1}}^{s_{2}} \frac{\partial \psi}{\partial s}(Q) d s
$$

for arbitrary $s_{1}, s_{2}$. Thus $(\partial \psi / \partial s)(Q)=0$ on $\lambda$ and hence on $\gamma_{0}$.

Lemma 4. Let $R, \gamma, Q_{0}, N\left(Q_{0}\right), N, \gamma_{0}$ be defined as in Lemma 3. Let $v(x, y)$ and $G(x, y)$ be of class $c^{0}$ on $N\left(Q_{0}\right)$ and of class $c^{1}$ on $N\left(Q_{0}\right)\left[R+Q_{0}\right]$. Let $v=G=0$ on $\gamma_{0}, \quad G>0$ in $R N\left(Q_{0}\right), \quad\left(\partial G / \partial_{\nu}\right)\left(Q_{0}\right) \neq 0$. Then there exists $\lim _{P \rightarrow Q_{0}} v(P) / G(P)$ for $P \in R$.

If $\gamma$ is of class $c^{k+1}$ on $N\left(Q_{0}\right)$ and $v$, $G$ are of class $c^{k}$ in $N\left(Q_{0}\right)$ and of class $c^{k+1}$ on $N\left(Q_{0}\right)\left[R+Q_{0}\right]$, then $v / G$ is of class $c^{k}$ on $N\left(Q_{0}\right)\left[R+Q_{0}\right]$.

Proof. Denote differentiation along a line parallel to $N$ by $\partial / \partial h$. By the mean value theorem one finds that $(\partial G / \partial \nu)\left(Q_{0}\right)$ is the limiting value of $(\partial G / \partial h)(P)$ as $P \in R N\left(Q_{0}\right)$ approaches $Q_{0}$ along the normal at $Q_{0}$, and hence $(\partial G / \partial \nu)\left(Q_{0}\right)$ is the limiting value of $(\partial G / \partial h)(P)$ as $P$ approaches $Q_{0}$ by any approach in $R N\left(Q_{0}\right)$. A similar statement is true for $\left(\partial v / \partial_{\nu}\right)\left(Q_{0}\right)$.

Let $P_{n}$ be any sequence of points in $R N\left(Q_{0}\right)$ converging to $Q_{0}$ and let $Q_{n}$ be the points on $\gamma_{0}$ associated, by projection along $N$, with $P_{n}$. By the generalized mean value theorem,

$$
\frac{v\left(P_{n}\right)}{G\left(P_{n}\right)}=\frac{v\left(P_{n}\right)-v\left(Q_{n}\right)}{G\left(P_{n}\right)-G\left(Q_{n}\right)}=\frac{(\partial v / \partial h)\left(P_{n}^{\prime}\right)}{(\partial G / \partial h)\left(P_{n}^{\prime}\right)}
$$

where $P_{n}^{\prime}$ is interior to the line segment $P_{n} Q_{n}$.

Thus

$$
\lim _{P_{n} \rightarrow Q_{0}} \frac{v\left(P_{n}\right)}{G\left(P_{n}\right)}=\frac{(\partial v / \partial \nu)\left(Q_{0}\right)}{(\partial G / \partial \nu)\left(Q_{0}\right)}
$$


It is clear from the construction of $N\left(Q_{0}\right)$ that the equations

$$
x=X(s, h)=x(s)+h \cos \alpha, \quad y=Y(s, h)=y(s)+h \cos \beta
$$

yield a one to one transformation of $N\left(Q_{0}\right)$ into $N^{*}\left(Q_{0}\right): 0 \leq h<h_{1}$, $0<s<L$ and $\gamma_{0}$ into $\gamma_{0}{ }^{*}: h=0,0<s<L$ and $Q_{0}$ into $Q_{0}{ }^{*}: h=0, s=s_{0}$. In fact, in view of the restriction on the slope of the tangent to $\gamma_{0}$, the Jacobian of the transformation is

$$
J=x^{\prime}(s) \cos \beta-y^{\prime}(s) \cos \alpha \neq 0 .
$$

If $x(s), y(s)$ are of class $c^{k+1}$ on $0<s<L$ then so are $X(s, h), Y(s, h)$ in $N^{*}\left(Q_{0}\right)-r_{0}{ }^{*}$. Any partial derivative of $X(s, h), \quad Y(s, h)$ of order $r \leq$ $k+1$ converges, as $h \rightarrow 0$, uniformly on any closed subinterval of $\gamma_{0}^{*}$ and thus this derivative has a continuous limit on $\gamma_{0}{ }^{*}$. By the implicit function theorems, the inverse functions $s=S(x, y), h=H(x, y)$ are of class $c^{k+1}$ in $R N\left(Q_{0}\right)$. Moreover, the partial derivatives of $S, H$ of order $r \leq k+1$ have continuous limits on $\gamma_{0}$, for the relationships

$$
\begin{aligned}
& 1=\frac{\partial X}{\partial s} \frac{\partial s}{\partial x}+\frac{\partial X}{\partial h} \frac{\partial h}{\partial x}=x^{\prime}(s) \frac{\partial s}{\partial x}+\cos \alpha \frac{\partial h}{\partial x} \\
& 0=\frac{\partial Y \frac{\partial s}{\partial s} \partial \frac{\partial Y}{\partial h} \partial h=y^{\prime}(s) \frac{\partial s}{\partial x}+\cos \beta \frac{\partial h}{\partial x}}{\partial h}
\end{aligned}
$$

can be solved for $\partial s / \partial x, \partial h / \partial x, \partial s / \partial y, \partial h / \partial y$ and the resulting equations indicate that these derivatives and their derivatives of order $\leq k$ have continuous limits on $\gamma_{0}$.

Under this transformation $v(x, y)$ becomes $v^{*}(s, h)$ and $G(x, y)$ becomes $G^{*}(s, h)$. It is sufficient to show $v^{*} / G^{*}$ is of class $c^{k}$ at $Q_{0}{ }^{*}$ since any partial derivative of order $r \leq k$ of $v(x, y) / G(x, y)$ is a polynomial in the derivatives of $v^{*} / G^{*}$ and in the derivatives of $s$ and $h$ with respect to $x$ and $y$ of order $\leq r$.

By the hypothesis and comments above, $v^{*}(s, h)$ and $G^{*}(s, h)$ are of class $c^{k}$ on $N^{*}(Q)$ and of class $c^{k+1}$ on $\left(N^{*}\left(Q_{0}\right)-r_{0}{ }^{*}\right)+Q_{0}{ }^{*}$. In view of the continuity of $\partial G / \partial h$ at $Q_{0}$. there is a neighborhood of $Q_{0}$ where $(\partial G / \partial h)(P)>\delta>0$, It is no loss of generality to assume $(\partial G / \partial h)>\delta>0$ in $N\left(Q_{0}\right)$ and we shall do so. By Lemma $3, \partial v / \partial s$ and $\partial G / \partial s$ vanish on $\gamma_{0}$. By repeated application of Lemma $3, \partial^{r} v / c s^{r}$ and $\partial^{r} G / \partial s^{r}(0 \leq r \leq k)$ vanish on $\gamma_{0}$.

The proof is greatly facilitated by an auxiliary transformation. Let $t=s, z=G^{*}(s, h)$ carrying $Q_{0}{ }^{*}$ into $Q_{0}{ }^{* *}, \gamma_{0}{ }^{*}$ into $\gamma_{0}{ }^{* *}, N^{*}\left(Q_{0}\right)$ into $N^{* *}\left(Q_{0}\right)$. For each $s, z$ is a monotone increasing function of $h$ and the inverse function $h=H^{*}(t, z)$ is a monotone increasing function of $z$ for each $t$. As above, we see that $v^{*}(s, h)=v^{* *}(t, z)$ is of class $c^{k}$ on 
$N^{* *}\left(Q_{0}\right)$ and of class $c^{k+1}$ on $\left(N^{* *}\left(Q_{0}\right)-\gamma_{0}{ }^{* *}\right)+\left(Q_{0}{ }^{* *}\right)$. Moreover, it suffices to prove that $v^{* *}(t, z) / z$ is of class $c^{k}$ at $Q_{0}{ }^{* *}$. For notational simplicity, let $w(t, z)=v^{* *}(t, z)$. Note that $N^{* *}\left(Q_{0}\right)$ is the set $0 \leq$ $z<G\left(t, h_{1}\right), 0<t<L$.

By induction, we verify

$$
\frac{\partial^{r}}{\partial z^{r}}\left(\frac{w}{z}\right)=\frac{r !(-1)^{r}}{z^{r+1}}\left(w-z \frac{\partial w}{\partial z}+\frac{z^{2}}{2 !} \frac{\partial^{2} w}{\partial z^{2}}+\cdots+(-1)^{r} \frac{z^{r} \partial^{r} w}{r ! \partial z^{r}}\right)
$$

for $0 \leq r \leq k$ when $z>0$.

For $t$ fixed, $w(t, z)$ has a Taylor expansion of the form

$$
\begin{aligned}
w(t, \zeta)= & w(t, z)+\frac{\partial w}{\partial z}(t, z)(\zeta-z)+\cdots+\frac{\partial^{r} w}{\partial z^{r}}(t, z) \frac{(\zeta-z)^{r}}{r !} \\
& +\frac{\partial^{r+1} w}{\partial z^{r+1}}(t, \xi) \frac{(\zeta-z)^{r+1}}{(r+1) !}
\end{aligned}
$$

for $0 \leq r \leq k$, where $0 \leq \zeta<\xi(t, z, \zeta, r)<z$ so that, when $\zeta=0$,

$$
\begin{aligned}
0=w(t, 0)= & w(t, z)-z \frac{\partial w}{\partial z}(t, z)+\cdots+\frac{(-1)^{r}}{r !} z^{r} \frac{\partial^{r} w}{\partial z^{r}}(t, z) \\
& +\frac{(-1)^{r+1} z^{r+1}}{(r+1) !} \frac{\partial^{r+1} w}{\partial z^{r+1}}(t, \xi) .
\end{aligned}
$$

Hence

$$
\frac{\partial^{r}}{\partial z^{r}}\left(\frac{w}{z}\right)=\frac{1}{r+1} \frac{\partial^{r+1}}{\partial z^{r+1}} w(t, \xi),
$$

which has a limit as the point $(t, z)$ approaches $Q_{0}{ }^{* *}$.

We have thus shown that the partial derivatives of $w / z$, with respect to $z$ alone, of order $\leq k$ have limits at $Q_{0}{ }^{* *}$.

We next show that the partial derivatives of $w / z$ with respect to $t$ alone have limits at $Q_{0}{ }^{* *}$. First note that the derivatives of $w$ with respect to $t$ alone vanish at $z=0$. For, $w(t, z)=v^{*}(s, h)$ so that

$$
\frac{\partial v}{\partial s}=\frac{\partial v^{*}}{\partial s}=\frac{\partial w}{\partial t}+\frac{\partial w}{\partial z} \frac{\partial z}{\partial s}=\frac{\partial w}{\partial t}+\frac{\partial w}{\partial z} \frac{\partial G}{\partial s}
$$

and, as we have seen, $\partial v / \partial s$ and $\partial G / \partial s$ vanish at $z=0$. Thus $\partial w / \partial t=0$ at $z=0$. Similarly, successive differentiation shows $\partial^{r} w / \partial t^{r}=0$ on $\gamma_{0}^{* *}$, $0 \leq r \leq k$.

We apply Taylor's theorem to obtain

$$
\begin{aligned}
\partial^{r}\left(\begin{array}{c}
w \\
z
\end{array}\right)=\frac{1}{z} \frac{\partial^{r} w(t, z)}{\partial t^{r}} & ={ }_{z}^{1}\left\{z \frac{\partial}{\partial z}\left[\frac{\partial^{r} w(t, \xi)}{\partial t^{r}}\right]\right\}=\frac{\partial}{\partial z} \frac{\partial^{r} w(t, \xi)}{\partial t^{r}}, \\
& 0<\xi(z, r)<z
\end{aligned}
$$


and conclude that $\left(\partial^{r} / \partial t^{r}\right)(w / z)$ has a limit at $Q_{0}{ }^{* *}$ for $0 \leq r \leq k$, Finally, any mixed derivative may be written as

$$
\partial z^{n} \partial t^{m}\left(\frac{w}{z}\right), \quad n+m=r \leq k
$$

and this may be written as $\begin{gathered}\partial^{n} \\ \partial z^{n}\end{gathered}\left\{\frac{1 \partial^{m} w}{z \partial t^{m}}\right\}$

where $\partial^{m} w / \partial t^{m}$ vanishes on $\gamma_{0}^{* *}$ and is of class $c^{k-m}$ on $N^{* *}\left(Q_{0}\right)$ and of class $c^{k-m+1}$ on $\left(N^{* *}\left(Q_{0}\right)-\gamma_{0}{ }^{* *}\right)+Q_{0}{ }^{* *}$. By the first results for derivatives with respect to $z$, the mixed derivatives have the desired property.

THEOREM 1. Let $R$ be a bounded region whose boundary I' consists of a finite number of non-intersecting simply closed regular arcs of class $c^{k},(k \geq 2)$. Let $G(x, y)$ be a function of class $c^{k}$ on $R+\Gamma$, vanishing on $\Gamma$, positive in $R$, with $\partial G / \partial \nu \geq \delta>0$ on $\Gamma$.

Let $H$ be the Hilbert space formed by completing the linear vector. space $V$ of variations - functions of class $c^{k}$ on $\bar{R}$ and vanishing on $\Gamma$-, using the functional

$$
D[\xi]=\iint\left(a \xi_{x}^{2}+b \xi_{y}^{2}+c \xi^{2}\right) d x d y
$$

for $\xi \in V$ as the square of the norm, where $a, b, c$ are bounded and integrable, $a>0, b>0, c \geq 0$ in $R+\Gamma$.

Then the set of functions $G \tau$, where $\tau$ is a polynomial in $x$ and $y$, is dense in $H$. The set $\left\{f_{i}\right\}$ obtained by orthonormalizing the set $\left\{G x^{i} y^{j}\right\}$ is complete in $H$.

If $g(x, y)$ is a function of class $c^{k}$ on $\bar{R}$ and $\Psi$ is the set of functions $\psi$ of class $c^{k}$ on $\bar{R}$, assuming the values of $g(x, y)$ on $\Gamma$, and if for any $\psi \in \Psi$ we define $b_{i}=D\left[\psi-g, f_{i}\right]$, then

$$
\left\|\psi-g-\sum_{i=1}^{n} b_{i} f_{i}\right\|^{2}=D\left[\psi-g-\sum_{i=1}^{n} b_{i} f_{i}\right] \leq \frac{\theta(n)}{n^{k-2}}
$$

where $\lim _{n \rightarrow \infty} \theta(n)=0, \theta$ depending on $\psi-g$.

In particular, if $f$ is integrable,

$$
I[\psi]=\iint\left(a \psi_{x}^{2}+b \psi_{y}^{2}+c \psi^{2}+2 f \psi^{\prime}\right) d x d y,
$$

and there exists an admissible function $\psi^{\prime \prime}$ which minimizes $I[\psi]$ for $\psi \in \Psi$, and we define 


$$
a_{i}=-\iint f f_{i} d x d y-D\left[g, f_{i}\right], \quad \phi_{n}=g+\sum_{i=1}^{n} a_{i} f_{i}
$$

then

$$
\left\|\psi_{0}-\phi_{n}\right\|^{2}=D\left[\psi_{0}-\phi_{n}\right] \leq \begin{gathered}
\theta(n) \\
n^{k-2}
\end{gathered}
$$

where $\lim _{n \rightarrow \infty} \theta(n)=0$.

Proof. If $v$ is a variation, we show there is a sequence $Q_{j}$ of polynomials such that

$$
\lim _{j \rightarrow \infty}\left\|v-G Q_{j}\right\|^{2}=\lim _{j \rightarrow \infty} \iint\left[a\left(v-G Q_{j}\right)_{x}^{2}+b\left(v-G Q_{j}\right)_{y}^{2}+c\left(v-G Q_{j}\right)^{2}\right] d x d y=0 .
$$

In view of Lemma $4, v / G$ is of class $c^{k-1}$ on $\bar{R}$ and it is thus possible to extend the definition of $v / G$ over the entire plane so that it is of class $c^{k-1}$ over the entire plane. Let $\Omega(\hat{\delta})$ be the uniform modulus of continuity of the $(k-1)$ st partial derivatives of $v / G$ over a rectangle with sides parallel to the axes containing $R$ in its interior.

By Lemma 2, with $s=2, j=1, m_{1}=m_{2}=j$ there is a sequence $Q_{j}$ of polynomials of degree $2 j$ in $x$ and $y$ such that, for $(x, y)$ in $\bar{R}$,

$$
\left|\frac{v}{G}-Q_{j}\right|, \quad\left|\left(\begin{array}{c}
v \\
G
\end{array}\right)_{x}-Q_{j_{x}}\right|, \text { and }\left|\left(\begin{array}{c}
v \\
G
\end{array}\right)_{y}-Q_{j_{y}}\right| \text { are all } O\left(\frac{1}{j^{k-2}} \Omega\left(\frac{1}{j}\right)\right) .
$$

Hence

$$
\begin{aligned}
\left(v-G Q_{j}\right)_{x}^{2}=\left[\left(\begin{array}{c}
v \\
G
\end{array}-Q_{j}\right) G\right]_{x}^{2}=\left[\left(\frac{v}{G}-Q_{j}\right)_{x} G+\left(\frac{v}{G}-Q_{j}\right) G_{x}\right]^{2} \\
\quad \leq\left(\begin{array}{c}
v \\
G
\end{array}-Q_{j}\right)_{x}^{2} G^{2}+G_{x}^{2}\left(\frac{v}{G}-Q_{j}\right)^{2}+2 G\left|G_{x}\right|\left(\begin{array}{c}
v \\
G
\end{array}-Q_{j}\right)_{x}|| \begin{array}{c}
v \\
G
\end{array}-Q_{j} \mid \\
=-O\left(\begin{array}{c}
1 \\
j^{2(k-2)}
\end{array}\left[\Omega\left(\begin{array}{l}
1 \\
j
\end{array}\right)\right]^{2}\right) .
\end{aligned}
$$

A similar result is true for $\left(v-G Q_{j}\right)_{y}^{2}$ and $\left(v-G Q_{j}\right)^{2}$. Thus $\lim _{j \rightarrow \infty} D[v$ $\left.-G Q_{j}\right]=0$ for $k \geq 2$.

It has thus been proved that the linear manifold formed by $\left\{G x^{i} y^{j}\right\}$ is dense in $V$ and thus in $H$. By the previous discussion the set $\left\{f_{i}\right\}$ is complete in $H$.

Now let $v$ in the above be the particular variation $\phi-g$ and let $[N]$ represent the largest integer $\leq N$. For fixed $n$, let $j=[(\sqrt{ } n / 2)-1]$ and $\tau_{n}(x, y)=Q_{j}(x, y)$. Thus there is a sequence $\tau_{\imath}$ of degree at most

$$
2 j \leq\left[2\left(\begin{array}{c}
\sqrt{ } n \\
2
\end{array}-1\right)\right]=[\sqrt{ } 2 n-2]
$$


such that

$$
D\left[\psi-g-G \tau_{n}\right]=O\left(\begin{array}{c}
1 \\
n^{k-2}
\end{array} \Omega^{2}\left(\begin{array}{c}
8 \\
V n
\end{array}\right)\right)
$$

Now $\sum_{i=1}^{n} b_{i} f_{i}=G \mu_{n}$ where $\mu_{n}$ is a polynomial of degree greater than $\sqrt{ } 2 n-2$, and it is known that

$$
\psi-g-\sum_{i=1}^{n} c_{i} f_{i}
$$

is a minimum when $c_{i}=\left(\psi-g, f_{i}\right)=b_{i}$. Thus

$$
D\left[\psi-g-\sum_{i=1}^{n} b_{i} f_{i}\right]=O\left(\begin{array}{c}
1 \\
n^{k-2}
\end{array}\right), \quad \lim _{n \rightarrow \infty} \theta=0 .
$$

In particular, if $\psi_{0}$ minimizes $I[\psi]$, then we have seen that

$$
D\left[\psi_{0}-g, f_{i}\right]=-\iint f f_{i} d x d y-D\left[g, f_{i}\right] .
$$

Thus, in this case, the Fourier coefficients depend only on known quantities.

CoROLlary

$$
b_{n}=O\left(\sqrt{ } \frac{\theta(n)}{n^{k-2}}\right) .
$$

$$
\begin{aligned}
\text { Proof: } b_{n=}^{2}= & D\left[b_{n} f_{n}\right]=D\left[\left(\psi-g-\sum_{i=1}^{n-1} b_{i} f_{i}\right)-\left(\psi-g-\sum_{i=1}^{n} b_{i} f_{i}\right)\right] \\
\leq & D\left[\psi-g-\sum_{i=1}^{n-1} b_{i} f_{i}\right]+2\left(D\left[\psi-g-\sum_{i=1}^{n-1} b_{i} f_{i}\right]\right. \\
& \left.\cdot D\left[\psi-g-\sum_{i=1}^{n} b_{i} f_{i}\right]\right)^{1 / 2}+D\left[\psi-g-\sum_{i=1}^{n} b_{i} f_{i}\right]=O\left(\frac{\theta(n)}{n^{k-2}}\right) .
\end{aligned}
$$

§3. Expansion Theorems. We use the notations in Theorem 1 and seek conditions which insure that convergence in $H$ yields uniform convergence in $\bar{R}$.

THEOREM 2. Let $R$ be a bounded region with boundary I'. Let $\psi^{\prime}, \psi_{n}$ be continuous on $\bar{R}$, absolutely continuous on each line in $\bar{R}$ and all taking on the same values on $\Gamma$. Let $D[\psi]<\infty, D\left[\psi_{n}\right]<\infty$. If $\lim _{n \rightarrow \infty} D\left[\psi-\psi_{n}\right]$ $=0$, then a necessary and sufficient condition that $\lim \psi_{n}=\stackrel{n \rightarrow \infty}{\psi}$ uniformly on $\bar{R}$ is that $\psi_{n}$ be equicontinuous on $\bar{R}$. If $\lim _{n, m \rightarrow \infty} \stackrel{n \rightarrow \infty}{D}\left[\psi_{n}-\psi_{m}\right]=0$ then $a$ necessary and sufficient condition that $\lim _{n \rightarrow \infty} \psi_{n}$ exists uniformly on $\bar{R}$ is that $\psi_{n}$ be equicontinuous on $\bar{R}$.

Proof. The necessity is clear since a sequence of continuous functions 
which converge uniformly are equicontinuous.

Let $u(x, y)$ be a function with the continuity properties of $\psi(x, y)$ and vanishing on $\Gamma$. Let $P_{0}$ be a point interior to $R$. Place polar coordinates at $P_{0}$. If a ray from $P_{0}$ meets the circle $S_{\rho}$ of radius $\rho \leq d$, $d$ being the diameter of $R$, with $P_{0}$ as center, before it meets $I$, label $P_{1}$ the first intersection point with $S_{\rho}$ and $Q$ the first intersection with $\Gamma$. Otherwise both $P_{1}$ and $Q$ will refer to the first intersection point of the ray and $\Gamma$.

$$
\begin{aligned}
& { }_{2 \pi}^{1}\left\{\int_{0}^{2 \pi}\left|u\left(P_{1}\right)\right| d \theta\right\}^{2} \leq \int_{0}^{2 \pi} u^{2}\left(P_{1}\right) d \theta=\int_{0}^{2 \pi}\left[\int_{P_{1}}^{Q} \partial r d r\right]^{2} d \theta \\
& =\int_{0}^{2 \pi}\left[\int_{P_{1}}^{Q} \frac{1}{V} \cdot V \cdot \cdot \frac{\partial u}{\partial r} d r\right]^{2} d \theta \leq \int_{0}^{2 \pi} \log \frac{d}{\rho} \int_{P_{1}}^{Q} r \cdot\left(\frac{\partial u}{\partial r}\right)^{2} d r \cdot d \theta \\
& \leq \log \frac{d}{\rho} \iint\left(u_{x}^{2}+u_{y}^{2}\right) d x d y \leq \alpha \log \frac{d}{\rho} D[u]
\end{aligned}
$$

where $\alpha=\frac{1 / \min _{R+F}}{(a, b)}$, since

$$
\iint\left(a u_{x}^{2}+b u_{y}^{2}+c \iota^{2}\right) d x d y \geq \min (a, b) \iint\left(u_{x}^{2}+u_{y}^{2}\right) d x d y .
$$

Apply this result to the functions $u_{n}=\psi-\psi_{n}$ (or to $u_{n m}=\psi_{n}-\psi_{m}$ ) which are equicontinuous on $R+I^{\prime}$ and thus have a uniform modulus of continuity $\omega(\delta)$, which approaches 0 with $\delta$.

Since $P_{1}$ is on or interior to the circle of radius $\rho$, we have $\left|u_{n}\left(P_{1}\right)-u_{n}\left(P_{0}\right)\right| \leq \omega(\rho)$, whence $\left|u_{n}\left(P_{1}\right)\right| \geq\left|u_{n}\left(P_{0}\right)\right|-\omega(\rho)$ and

$$
2 \pi\left[\left|u_{n}\left(P_{0}\right)\right|-\omega(\rho)\right] \leq \sqrt{2 \pi \alpha \log d / \rho D\left[u_{n}\right]} .
$$

Thus

$$
\left|u_{n}\left(P_{0}\right)\right| \leq \sqrt{\frac{\alpha}{2 \pi} D\left[u_{n}\right] \log \frac{d}{\rho}+\omega(\rho)}
$$

which is true even if $P_{0}$ is on $\Gamma$.

Now, for $\varepsilon>0$, choose $\rho=\rho_{1}$ so small that $\omega\left(\rho_{1}\right)<\varepsilon / 2$ and then choose $N$ so large that

$$
\underset{2 \pi}{\alpha} D\left[u_{n}\right] \log \frac{d}{\rho_{1}}<\frac{\varepsilon^{2}}{4}
$$

for $n>N$. Hence

$$
\varepsilon>0 \cdot \supset \cdot \sharp N(\varepsilon) \ni n>N \cdot \supset \cdot\left|\psi\left(P_{0}\right)-\psi_{n}\left(P_{0}\right)\right|<\varepsilon .
$$

Lemma 5. Let $R$ be a bounded region with boundary $I$ and diameter $d$. 
Let $u(x, y)$ be continuous on $R+\Gamma$, absolutely continuous on each line in $R+I^{\prime}$, and vanish on $I^{\prime}$, and let $0<D[u]<\infty$. Let $\alpha=1 / \min _{n+r}(a, b)$. Let $P_{0} \in R+\Gamma$. If there exists $\delta>0, K \geq 0$ and

$$
\left|u(P)-u\left(P_{0}\right)\right| \leq K\left\|P-P_{0}\right\|^{\$}
$$

for all points $P$ such that the ray $P_{0} P$ is in $R+\Gamma$, then

$$
\left|u\left(P_{0}\right)\right| \leq \sqrt{\frac{\alpha D[u]}{2 \pi \delta} \log ^{+}-\frac{d^{\delta} K}{\Delta D[u]}}+\Delta D[u]
$$

where $\Delta$ is any number $>0$, and

$$
\log ^{+} x=\left\{\begin{array}{ccc}
\log x & \text { if } & x>1 \\
0 & \text { if } & x \leq 1
\end{array}\right.
$$

Proof. If $P_{0}$ is interior to $R$, and $\rho \leq d$, then as in Theorem 2

$$
\frac{1}{2 \pi}\left\{\int_{0}^{2 \pi}\left|u\left(P_{1}\right)\right| d \theta\right\}^{2} \leq \alpha \log d / \rho D[u],
$$

where $P_{1}$ is a point which is the first intersection of a ray from $P_{\|}$ with either $\Gamma$ or the circle of radius $\rho \leq d$ about $P_{0}$ as center.

Since $P_{1}$ is on or interior to the circle of radius $\rho$, we have

$$
\begin{gathered}
\left|u\left(P_{1}\right)-u\left(P_{0}\right)\right| \leq K \rho^{\delta}, \quad\left|u\left(P_{1}\right)\right| \geq\left|u\left(P_{0}\right)\right|-K \rho^{\delta}, \\
2 \pi\left[u\left(P_{0}\right)-K \rho^{\delta}\right] \leq \sqrt{2 \pi \alpha} \log d \mid \rho D[u], \\
\left|u\left(P_{0}\right)\right| \leq \sqrt{\frac{\alpha}{2 \pi} D[u] \log } \frac{d}{\rho}+K \rho^{\delta},
\end{gathered}
$$

which holds even if $P_{0}$ is on $\Gamma$.

Let $\Delta>0$. If

$$
\left(\frac{\Delta D[u]}{K}\right)^{1 / \delta}<d
$$

choose

$$
\rho=\left(\frac{\Delta D[u]}{K}\right)^{1 / \delta}
$$

to obtain

$$
\left|u\left(P_{0}\right)\right| \leq \sqrt{\frac{\alpha}{2 \pi \delta} D[u] \log \frac{d^{\delta} K}{\Delta D[u]}}+\Delta D[u] .
$$

Otherwise,

$$
\left(\frac{\Delta D[u]}{K}\right)^{1 / \delta} \geq d, \quad K \leq^{\Delta} \frac{D[u]}{d^{\delta}}
$$


and we may replace $K$ to obtain

$$
\left|u\left(P_{0}\right)\right| \leq \sqrt{2 \pi} \frac{\alpha}{-} D[u] \log \frac{d}{\rho}+\frac{\Delta D[u]}{d^{\delta}} \rho^{\delta} .
$$

Choose $\rho=d$ to obtain $\left|u\left(P_{0}\right)\right| \leq \Delta D[u]$.

Corollary 1. A sufficient condition that a sequence $u_{n}$, absolutely continuous on each line in $\bar{R}$, vanishing on $\Gamma$, continuous on $\bar{R}$, and having $\lim D\left[u_{n}\right]=0$, converge to 0 at $P_{0}$ is that $\exists \delta>0$ and a sequence $K_{n}$, with $\lim _{n \rightarrow \infty}^{n} D\left[u_{n}\right] \log K_{n}=0$ such that

$$
\left|u_{n}(p)-u_{n}\left(P_{0}\right)\right| \leq K_{n}\left\|P-P_{0}\right\|^{\delta}
$$

for all $P$ with ray $P_{0} P$ in $\bar{R}$. If $\delta, K_{n}$ are independent of $P_{0}$, the convergence is uniform. In any case,

$$
\left|u_{n}\left(P_{0}\right)\right| \leq \sqrt{\frac{\alpha}{2 \pi \delta} D\left[u_{n}\right] \log ^{+} \frac{d^{\delta} K_{n}}{\Delta_{n} D\left[u_{n}\right]}+\Delta_{n} D\left[u_{n}\right]}
$$

for any $\Delta_{n}>0$.

Lemma 6. Let $R$ be a bounded domain with boundary $\Gamma$. Let $P_{0} \in \bar{R}$ and suppose there is a circle of radius $\varepsilon$ lying in $\bar{R}$ and containing $P_{0}$. Place polar coordinates $(r, \theta)$ at $P_{0}$. Let $u(x, y)$ be of class $c^{1}$ in $\bar{R}$ and suppose that there exist $\lambda>0, \sigma \geq 0$ such that

$$
\left|u_{r}(P)-u_{r}\left(P_{0}\right)\right| \leq \sigma\left\|P-P_{0}\right\|^{\lambda}
$$

for all points $P$ such that the ray $P_{0} P$ is in $\bar{R}$.

Then

$$
\begin{array}{ll}
\left.\operatorname{lv} u\left(P_{0}\right)\right|^{2} \leq\left(\sigma^{2} D[u]^{\lambda}\right)^{1 /(\lambda+1)}\left(\begin{array}{c}
\alpha \\
\lambda \pi
\end{array}\right)^{\lambda /(\lambda+1)} 2^{(5 \lambda+3) /(\lambda+1)}(\lambda+1)+\underset{\pi \varepsilon^{2}}{8 \alpha D[u]}\left(\frac{\lambda+1}{\lambda}\right) . \\
\text { Proof. } & \left|u_{r}\left(P_{0}\right)\right| \leq\left|u_{r}(P)\right|+\sigma r^{\lambda}
\end{array}
$$

Integrating over a circle $S_{\rho}$ of radius $\rho \leq \varepsilon$ which contains $P_{0}, S_{\rho} \subset$ $S_{z}$, we obtain

$$
\iint_{S_{\rho}}\left|u_{r}\left(P_{0}\right)\right|^{2} r d r d \theta \leq 2 \iint_{S_{\rho}} u_{r}(P)^{2} r d r d \theta+2 \iint_{S_{\rho}} \sigma^{2} r^{2 \lambda} r d r d \theta .
$$

We may assume that the polar axis lies in the direction of $\nabla u\left(P_{0}\right)$. Hence $u_{r}\left(P_{0}\right)=\left|\nabla u\left(P_{n}\right)\right| \cos \theta$ and

$$
\iint_{S_{p}}\left|\nabla u\left(P_{0}\right)\right|^{2}\left(\cos ^{2} \theta\right) r d r d \theta<2 \alpha D[u]+2 \sigma^{2}(2 \rho)^{2 \lambda} \cdot \pi \mu^{2}
$$


We will show that the minimum value of $\iint_{S_{\rho}}\left(\cos ^{2} \theta\right) r d r d \theta$ is $\pi \rho^{2} / 4$. Suppose first that the pole $O$ is interior to $S_{\rho}$. Let $r(\theta)$ be the equation of the circle relative to the pole $O$. Let $Q$ be the point $(r(\theta), \theta)$ and $Q^{\prime}$ the point $(r(\theta+\pi), \theta+\pi) . Q$ and $Q^{\prime}$ are thus the intersections of a ray through $O$ with the circle. Let $O^{\prime}$ be the center of the circle and suppose the coordinates of $O^{\prime}$ relative to $O$ are $(c, \phi)$. Then the angle between $O Q$ and $O O^{\prime}$ is $\phi-\theta$. Drop a perpendicular from $O^{\prime}$ to $Q O Q^{\prime}$ hitting the latter at $T$, the length of $O T$ being $|c \cos (\phi-\theta)|$. Thus one of

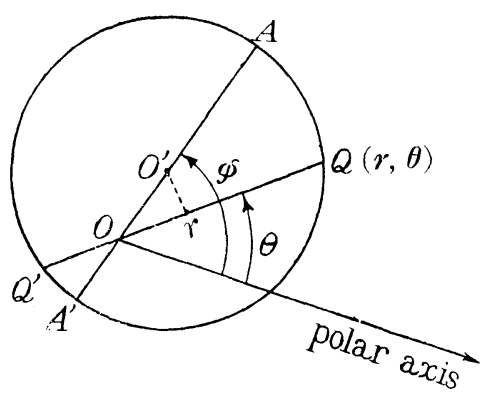
the lengths $\|O Q\|,\left\|O Q^{\prime}\right\|$ is $m+|c \cos (\phi-\theta)|$ and the other is $m-$ $|c \cos (\phi-\theta)|$ where $2 m$ is the length of $Q Q^{\prime}$, and the product $\left\|O Q^{\prime}\right\|$ - $\|Q Q\|=m^{2}-c^{2} \cos ^{2}(\phi-\theta)$. Also, if $O O^{\prime}$ meets the circle in points $A$, $A^{\prime}$ it is easily seen that $\left\|O A^{\prime}\right\|\|O A\|=\|O Q\|\left\|O Q^{\prime}\right\|$ so that $(\rho+c)(\rho-c)$ $=m^{2}-c^{2} \cos ^{2}(\phi-\theta)$ and $m^{2}=\rho^{2}-c^{2}+c^{2} \cos ^{2}(\phi-\theta)$. Hence

$$
\begin{aligned}
\|O Q\|^{2}+\left\|O Q^{\prime}\right\|^{2} & =[m+|c \cos (\phi-\theta)|]^{2}+[m-|c \cos (\phi-\theta)|]^{2} \\
& =2 m^{2}+2 c^{2} \cos ^{2}(\phi-\theta)=2 \rho^{2}-2 c^{2}+4 c^{2} \cos ^{2}(\phi-\theta) .
\end{aligned}
$$

We note that

$$
\begin{aligned}
\iint_{S_{\rho}}\left(\cos ^{2} \theta\right) r d r d \theta & =\frac{1}{2} \int_{0}^{2 \tau} r^{2}(\theta) \cos ^{2} \theta d \theta=\frac{1}{2} \int_{0}^{\pi}\left(\|O Q\|^{2}+\left\|O Q^{\prime}\right\|^{2}\right) \cos ^{2} \theta d \theta \\
& =\frac{1}{2} \int_{0}^{\pi}\left[2 \rho^{2}-2 c^{2}+4 c^{2} \cos ^{2}(\phi-\theta)\right] \cos ^{2} \theta d \theta .
\end{aligned}
$$

Moreover this formula holds even if $O$ is a point on the circumference for in this case

$$
\iint_{s_{p}}\left(\cos ^{2} \theta\right) r d r d \theta=1 / 2 \int_{\gamma}^{\gamma+\pi} r^{2}(\theta) \cos ^{2} \theta d \theta
$$

where $r$ is the angle between the polar axis and the tangent to the circle at $O$ in that direction which has the area to the left of the tangent line. Here $r^{2}=[2 \rho \cos (\phi-\theta)]^{2}$ and since the square of the cosine has period $\pi$, the integral reduces to

$$
\frac{1}{2} \int_{0}^{\pi} 4 \rho^{2} \cos ^{2}(\phi-\theta) \cos ^{2} \theta d \theta \text {. }
$$

Thus, in any case, 


$$
\begin{aligned}
\iint_{S_{\mathrm{p}}}\left(\cos ^{2} \theta\right) r d r d \theta & =\frac{1}{2} \int_{0}^{\pi}\left[2 \rho^{2}-2 c^{2}+4 c^{2} \cos ^{2}(\phi-\theta)\right] \cos ^{2} \theta d \theta \\
& =\frac{1}{2} \pi \rho^{2}-\frac{1}{2} \pi c^{2}+\frac{\pi c^{2}}{4}\left[1+2 \cos ^{2} \phi\right] .
\end{aligned}
$$

For fixed $c$, the minimum is obtained when $\phi=\frac{\pi}{2}$, and is $\begin{gathered}\pi \rho^{2} \\ 2\end{gathered}-\frac{1}{4} \pi c^{2}$. The absolute minimum is obtained when $c=\rho$ and is $\pi \rho^{2} / 4$.

It follows from this result, that

$$
\begin{gathered}
\frac{\pi \rho^{2}}{4}\left|\nabla u\left(P_{0}\right)\right|^{2} \leq 2 \alpha D[u]+2 \sigma^{2}(2 \rho)^{2 \lambda} \pi \rho^{2}, \\
\left|\nabla u\left(P_{0}\right)\right|^{2}<\frac{8 \alpha \underset{\pi \rho^{2}}{D}[u]}{\pi \rho^{2 \lambda+3} \sigma^{2} \rho^{2 \lambda}} .
\end{gathered}
$$

Consider the function $y=A / \rho^{2}+B \rho^{2 \lambda}$ where $A=8 \alpha D[u] / \pi, B=2^{2 \lambda+3} \sigma^{2}$. The minimum value is

$$
\begin{aligned}
y_{\mathrm{min}} & =A^{\lambda /(\lambda+1)} B^{1 /(\lambda+1)}(\lambda+1) \lambda^{-\lambda /(\lambda+1)} \\
& =\left(\sigma^{2} D[u]^{\lambda}\right)^{1 /(\lambda+1)}\left(\begin{array}{l}
\alpha \\
\lambda \pi
\end{array}\right)^{\lambda /(\lambda+1)}(\lambda+1) 2^{(5 \lambda+3) /(\lambda+1)}
\end{aligned}
$$

obtained when

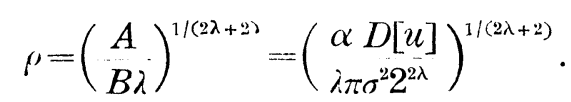

If

$$
\left(\frac{\alpha D[u]}{\lambda \pi \sigma^{2} 2^{2 \lambda}}\right)^{1 /(2 \lambda+2)}<\varepsilon
$$

choose

$$
\rho=\left(\begin{array}{c}
\alpha D[u] \\
\lambda \pi \sigma^{2} 2^{2 \lambda}
\end{array}\right)^{1 /(2 \lambda+2)}
$$

and have

$$
\left|\nabla u\left(P_{0}\right)\right|^{2} \leq\left(\sigma^{2} D[u]^{\lambda}\right)^{1 /(\lambda+1)}\left(\frac{\alpha}{\pi \lambda}\right)^{\lambda /(\lambda+1)} 2^{(5 \lambda+3) /(\lambda+1)}(\lambda+1) .
$$

However, if

$$
\left(\frac{\alpha D[u]}{\lambda \pi \sigma^{2} 2^{2 \lambda}}\right)^{1 /(2 \lambda+2)}>\varepsilon
$$

we have

$$
\sigma^{2} \leq \frac{\alpha D[u]}{2^{2 \lambda} \lambda \pi \varepsilon^{2 \lambda+2}}
$$


and integrating over $S_{\varepsilon}$, as in the beginning of this proof, we find that

$$
\begin{gathered}
\iint_{S_{\varepsilon}}\left|u_{r}\left(P_{0}\right)\right|^{2} r d r d \theta \leq 2 \iint_{\varsigma_{\varepsilon}} u_{r}(P)^{2} r d r d \theta+2 \frac{\alpha D[u]}{2^{2 \lambda} \lambda \pi \varepsilon^{2 \lambda+2}}(2 \varepsilon)^{2 \lambda} \pi \varepsilon^{2}, \\
\pi \varepsilon^{2}\left|\nabla u\left(P_{0}\right)\right|^{2} \leq 2 \alpha D[u]+\frac{2 \alpha D[u]}{\pi \lambda \varepsilon^{2}} \cdot \pi \varepsilon^{2}, \\
\left|\nabla u\left(P_{0}\right)\right|^{2} \leq \frac{8 \alpha D[u]}{\pi \varepsilon^{2}}+\frac{8 \alpha D[u]}{\lambda \pi \varepsilon^{2}}=\left(1+\frac{1}{\lambda}\right) \frac{8 \alpha D[u]}{\pi \varepsilon^{2}} .
\end{gathered}
$$

Thus, in any case,

$$
\left|\nabla u\left(P_{0}\right)\right|^{2} \leq\left(\sigma^{2} D[u]^{\lambda}\right)^{1 /(\lambda+1)}\left(\begin{array}{c}
\alpha \\
\lambda \pi
\end{array}\right)^{\lambda /(\lambda+1)} 2^{(i \lambda+3) /(\lambda+1)}(\lambda+1)+\frac{8 \alpha D[u]}{\pi \varepsilon^{2}}\left(\frac{\lambda+1}{\lambda}\right) .
$$

Lemma 7. Let $R$ be a bounded region with boundary $\Gamma$ and diameter $d$ and let $R$ have the property that there exists an $\varepsilon>0$ such that every point of $R+\Gamma$ is within some circle of radius $\varepsilon$ lying in $R+\Gamma$.

Let $u(x, y)=G \tau+H$ where $\tau$ is a polynomial of degree $m, G$ and $H$ are of class $c^{1}$ on $R+\Gamma$ and vanish on $\Gamma, G>0$ in $R,|\nabla G| \geq \delta>0$ on $\Gamma$. Let $|G| \leq G_{1},|H| \leq H_{1},|\nabla G| \leq G_{2},|\nabla H| \leq H_{2}$ for constants $G_{1}, G_{2}, H_{1}, H_{2}$.

Suppose also that

$$
\left|G_{x}(P)-G_{x}\left(P_{0}\right)\right| \leq G_{0}\left\|P-P_{0}\right\|, \quad\left|G_{y}(P)-G_{y}\left(P_{0}\right)\right| \leq G_{0}\left\|P-P_{0}\right\|,
$$

$\left|H_{x}(P)-H_{x}\left(P_{0}\right)\right| \leq H_{0}\left\|P-P_{0}\right\|, \quad\left|H_{y}(P)-H_{y}\left(P_{0}\right)\right| \leq H_{0}\left\|P-P_{0}\right\|$ for constants $G_{0}, H_{0}$, whenever $P, P_{0}$ are points in $\bar{R}$ such that the line $P_{0} P$ is in $\bar{R}$. Let $A$ be an upper bound for $D[u]$ and $D[u] \log m$.

Then there exists a constant $B$, depending only on $\alpha, A, G_{0}, G_{1}, G_{2}, H_{0}$, $H_{1}, H_{2}, \delta, \varepsilon, d, G$ but not on $m$ or $\tau$, such that for $P_{0} \in \bar{R}$.

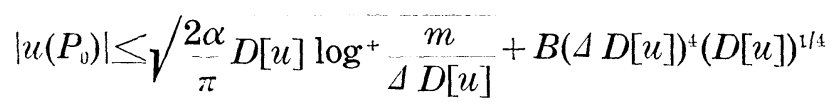

for any $\Delta>0$. ( $m$ to be replaced by 1 if it is 0$)$.

Proof. We may assume $D[u]>0$ for otherwise $u=0$ in $\bar{R}$.

Let $L=\max _{\bar{L}}|\tau|$. By a theorem of Kellogg [3], $|\nabla \tau(P)| \leq L m^{2} / \varepsilon$ for $P \in \bar{R}$.

If $P$ and $P_{0}$ are on a straight line in $\bar{R}$, then

$$
\begin{gathered}
\left|\tau(P)-\tau\left(P_{0}\right)\right|=\left|\int_{P_{0}}^{P^{P}} \frac{\partial \tau}{\partial r} d r\right| \leq \frac{L m^{2}}{\varepsilon}\left\|P-P_{0}\right\| . \\
\left|H(P)-H\left(P_{0}\right)\right| \leq H_{2}\left\|P-P_{0}\right\|, \quad\left|G(P)-G\left(P_{0}\right)\right| \leq G_{2}\left\|P-P_{0}\right\|, \\
\left|u(P)-u\left(P_{0}\right)\right| \leq\left|G(P) \tau(P)-G(P) \tau\left(P_{0}\right)\right|+\left|G(P)_{\tau}\left(P_{0}\right)-G\left(P_{0}\right) \tau\left(P_{0}\right)+\right| H(P)-H\left(P_{0}\right) \mid
\end{gathered}
$$




$$
\leq\left(G_{1} \frac{L m^{2}}{\varepsilon}+L G_{2}+H_{2}\right)\left\|P-P_{0}\right\|=K\left\|P-P_{0}\right\|
$$

By Lemma 5 , with $\Delta=D[u]^{-1 / 2}$,

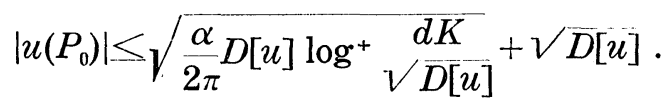

Also, $\tau_{x}, \tau_{y}$ are polynomials of degree $m$ and absolute value less than or equal to $L m^{2} / \varepsilon$, so that $\left|\nabla \tau_{x}\right| \leq\left(L m^{2} / \varepsilon\right)\left(m^{2} / \varepsilon\right.$, ) and

$$
\left|\tau_{x}(P)-\tau_{x}\left(P_{0}\right)\right| \leq \int_{P_{0}}^{P}\left|\nabla \tau_{x}\right| d r \leq \frac{L m^{4}}{\varepsilon^{2}}\left\|P-P_{0}\right\|
$$

Thus

$$
\left|\nabla \tau(P)-\nabla \tau\left(P_{0}\right)\right| \leq \frac{2 L m^{4}}{\varepsilon^{2}}\left\|P-P_{0}\right\|
$$

Then

$\left|\nabla u(P)-\nabla u\left(P_{0}\right)\right|$

$$
\begin{aligned}
& \leq\left|G(P)_{\nabla \tau}(P)-G\left(P_{0}\right) \nabla \tau\left(P_{0}\right)\right|+\left|\tau(P)_{\nabla} G(P)-\tau\left(P_{0}\right)_{\nabla} G\left(P_{0}\right)\right|+\left|\nabla H(P)-\nabla H\left(P_{0}\right)\right| \\
& \leq\left|G(P)_{\nabla \tau}(P)-G(P)_{\nabla \tau}\left(P_{0}\right)\right|+\left|G(P)_{\nabla \tau}\left(P_{0}\right)-G\left(P_{0}\right) \nabla \tau\left(P_{0}\right)\right| \\
& \quad+\left|\tau(P)_{\nabla} G(P)-\tau(P)_{\nabla} G\left(P_{0}\right)\right|+\left|\tau(P)_{\nabla} G\left(P_{0}\right)-\tau\left(P_{0}\right) \nabla G\left(P_{0}\right)\right| \\
& \quad+\left|H_{x}(P)-H_{x}\left(P_{0}\right)\right|+\left|H_{y}(P)-H_{y}\left(P_{0}\right)\right| \\
& \leq \\
& \quad\left(G_{1} \frac{2 L m^{4}}{\varepsilon^{2}}+\frac{L m^{2}}{\varepsilon} G_{2}+L 2 G_{0}+G_{2} \frac{L m^{2}}{\varepsilon}+2 H_{0}\right)\left\|P-P_{0}\right\|=\sigma\left\|P-P_{0}\right\| .
\end{aligned}
$$

Whence Lemma 6 yields

$$
\left|\nabla u\left(P_{0}\right)\right| \leq \sqrt{\left(\sigma^{2} D[u]\right)^{1 / 2}\left(\frac{\alpha}{\pi}\right)^{1 / 2} 32}+\frac{16 \alpha D[u]}{\pi \varepsilon^{2}} .
$$

By use of inequalities (2) and (3) we now find a bound for $L$.

Either $L \leq 1$ or else there exist constants $c_{1}, c_{2}$ such that $K<$ $c_{1} L m^{2}, \sigma<c_{2} L m^{4}$ where the factor $m$ is to be omitted if it is zero, and $c_{1}, c_{2}$ depend only on $\varepsilon, G_{1}, G_{2}, H_{2}, H_{0}, G_{0}$.

Assume $L>1$. Since $|\nabla G| \neq 0$ on $\Gamma$, there exists a continuous curve (or curves) $\gamma$ dividing $\bar{R}$ into two closed sets $\bar{R}_{1}$ and $\bar{R}_{2}$, such that $\bar{R}_{1} \bar{R}_{2}=\gamma, \bar{R}_{1}$ being a boundary set where $|\nabla G| \geq \delta / 2>0$, and $\bar{R}_{2}$ the set separated from $\Gamma$ by $\gamma$. There is a constant $c_{3}$ such that $G(P)$ $\geq c_{3}>0$ for $P \in \overline{R_{2}}$.

Suppose first that $|\tau|$ assumes its maximum $L$ at a point $P_{0} \in \vec{R}_{2}$. Then, by (2),

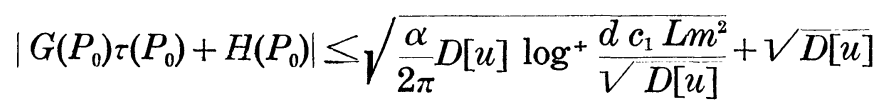


or,

$$
L \leq{ }_{c_{3}}^{1}\left[H_{1}+\sqrt{{ }_{2 \pi}^{\alpha} D[u] \log ^{+}} \frac{d c_{1} L m^{2}}{\sqrt{D} D[u]}+V D[u]\right] .
$$

Since $D[u] \log m$ and $D[u]$ are bounded by $A$, equation (4) implies the existence of a constant $c_{4}$ depending on $c_{3}, c_{1}, d, A, \alpha, H_{1}$ such that $L<c_{4}$.

On the other hand, if $|\tau|$ assumes its maximum $L$ at a point $P_{0} \in$ $\bar{R}_{1}$, write

$$
\begin{aligned}
& \nabla u=G \nabla \tau+\tau \nabla G+\nabla H \quad \tau^{2} \nabla G=\tau \nabla u-u \nabla \tau+H \nabla \tau-\tau \nabla H \\
& \tau^{2}|\nabla G| \leq|\tau||\nabla u|+|u||\nabla \tau|+|H||\nabla \tau|+|\tau||\nabla H| \\
& \leq L \sqrt{ }\left(c_{2}^{2} L^{2} m^{8} D[u]\right)^{1 / 2}\left(\begin{array}{c}
\alpha \\
\pi
\end{array}\right)^{1 / 2} 32+\frac{16 \alpha D[u]}{\pi \varepsilon^{2}} \\
&+\frac{L m^{2}}{\varepsilon}\left(\sqrt{2 \pi}_{2 \pi}^{\alpha} D[u] \log ^{+} \frac{d c_{1} L m^{2}}{V D[u]}+\sqrt{D[u]}\right)+H_{1} \frac{L m^{2}}{\varepsilon}+L H_{2} .
\end{aligned}
$$

Therefore,

$$
\begin{aligned}
L \leq & \frac{2}{\delta}\left\{\sqrt{32\left(c_{2}^{2} L^{2} m^{8} D[u]\right)^{1 / 2}\left(\begin{array}{c}
\alpha \\
\pi
\end{array}\right)^{1 / 2}+\frac{16 \alpha D[u]}{\pi \varepsilon^{2}}}\right. \\
& +\frac{m^{2}}{\varepsilon}\left(\sqrt{\left.\left.\frac{\alpha D[u]}{2 \pi} \log ^{+} \frac{d c_{1} m^{2}}{V \bar{D}[u]}+\sqrt{D[u]}\right)+\frac{H_{1} m^{2}}{\varepsilon}+H_{2}\right\}} .\right.
\end{aligned}
$$

This inequality, which is of the form

$$
L \leq K_{1}+K_{2} m^{2}+K_{3} m^{2} V \log L+K_{4} m^{2} V \dot{L},
$$

shows that

$$
\sqrt{L} \leq \frac{K_{1}}{\sqrt{L}}+\frac{K_{2} m^{2}}{\sqrt{L}}+K_{3} m^{2} \sqrt{\log L} \underset{L}{L}+K_{4} m^{2} \leq K_{1}+K_{2} m^{2}+K_{3} m^{2}+K_{4} m^{2},
$$

since $L>1$, whence $L \leq c_{5} m^{4}$ for some constant $c_{5}$.

Thus, in any case, there is a constant $c_{6}$ such that $L<c_{6} m^{4}$, where the factor $m$ is to be omitted if it is zero. From this one can conclude that $K<c_{1} c_{6} m^{6}$. However, we may obtain a better estimate by noticing that $K$ merely serves as a number such that $\left|u(P)-u\left(P_{0}\right)\right|$ $\leq K\left\|P-P_{0}\right\|$ whenever $P$ and $P_{0}$ are on a straight line in $\bar{R}$. Hence $K$ may be replaced by $\sup _{-}|\nabla u|$.

The inequality $\sigma<c_{2} \stackrel{\bar{R}}{L} m^{4}<c_{2} c_{6} m^{8}$ and formula (3) yield

$$
\left.\left|\nabla u\left(P_{0}\right)\right| \leq \sqrt{c_{2} c_{6} m^{8} D[u]^{1 / 2}\left(\frac{\alpha}{\pi}\right.}\right)^{1 / 2} \cdot 32+\frac{16 \alpha D[u]^{1 / 2} D[u]^{1 / 2}}{\pi \varepsilon^{2}}=c_{7} D[u]^{1 / 4} m^{4},
$$


since $D[u] \leq A$. Thus we may replace $K$ by $c_{7} D[u]^{1 / 4} m^{4}$ and substitute in Lemma 5 to obtain

$$
\left|u\left(P_{0}\right)\right| \leq \sqrt{2 \pi} \frac{{ }_{2 \pi}^{\alpha} D[u] \log +\frac{d c_{7} D[u]^{1 / t} m^{2}}{\Delta D[u]}}{\Delta D}+\Delta D[u] .
$$

Let $A=d c_{\tau} \Delta_{1}^{4} D[u]^{13 / 4}$ and $B=d c_{7}$ to obtain the conclusion.

Lemma 8. Let $R, G$, have the properties in Lemma 7 and let $u=$ $G \tau$ where $\tau$ is a polynomial of degree $m$.

Then $D[u] \geq c_{12}\left|u\left(P_{0}\right)\right|^{2} / \log m$ where $c_{12}>0$ is a constant depending only on $G_{0}, G_{1}, G_{2}, d, \varepsilon, \alpha, \delta, G$. The factor $\log m$ is to be omitted if $m=0$ or 1 .

Proof. Whether $L \leq 1$ or not, the formulas for $K$, $\sigma$ show that $K<c_{1} L m^{2}, \sigma<c_{2} L m^{4}$. Moreover, either formula (4) or (5) holds, with $H_{1}=0, H_{2}=0$. If (4) holds, we have

$$
\frac{L}{\sqrt{D[u]}} \leq \frac{1}{c_{3}}\left[\sqrt{\frac{\alpha}{2 \pi} \log ^{+} \frac{d c_{1} L m^{2}}{V D[u]}}+1\right] \text {. }
$$

Let $w=L / \sqrt{D[u]}$. The above inequality is then of the form $w \leq$ $K_{1} \sqrt{ } \log w m^{2}+K_{2}$ whence $L / \sqrt{D[u]} \leq c_{8} \log m$ for some constant $c_{8}$, depending on $\alpha, c_{3}, d, c_{1}$. Here the factor $\log m$ is to be omitted if $m=0$ or 1. On the other hand, if (5) holds, we have

$$
\begin{aligned}
\frac{L}{\sqrt{D[u]}} \leq \frac{2}{\delta}\left\{\sqrt{c_{2} m^{4}} \frac{L}{\sqrt{D[u]}}\left(\frac{\alpha}{\pi}\right)^{1 / 2} \cdot 32+\sqrt{\frac{16 \alpha}{\pi \varepsilon^{2}}}\right. \\
\left.+\frac{m^{2}}{\varepsilon}\left(\sqrt{\frac{\alpha}{2 \pi} \log ^{+} d c_{1} m^{2}-\frac{L}{V D[u]}}+1\right)\right\},
\end{aligned}
$$

from which we conclude $L / V D[u] \leq c_{9} m^{4} \log m$ ( $m$ and $\log m$ to be omitted if $m=0$ or 1 ).

Thus, in any case, there is a constant $c_{10}$ such that $L / V D[u] \leq$ $c_{10} m^{5}$. Therefore

$$
\underset{\sqrt{D[u]}}{K} \leq \frac{c_{1} L m^{2}}{\sqrt{D[u]}} \leq c_{1} c_{10} m^{7}
$$

Substituting in equation (2), we have

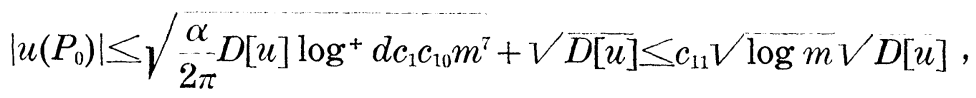

$m$ to be omitted if it is 0 or 1 . 
Theorem 3. Let $R$ be a hounded region whose boundary $I$ consists of a finite number of simply closed regular arcs of class $c^{k}, k \geq 3$. Let $G(x, y)$ be a function of class $c^{k}$ on $R+\Gamma$, vanishing on $I$, positive in $R$, with $\partial G / \partial \nu \geq \delta>0$ on $\Gamma$. Let $f_{i}$ be the set obtained by orthonormalizing the set $\left\{G x^{i} y^{j}\right\}$ using the functional

$$
D[\xi]=\iint_{R}\left(a \xi_{x}^{2}+b \xi_{y}^{2}+c \xi^{2}\right) d x d y
$$

as the square of the norm, where $a, b, c$ are bounded and integrable, $a>0, b>0, c \geq 0$ on $R+\Gamma$. Let $g(x, y)$ be any function of class $c^{k}$ on $R+\Gamma$. Let $\psi(x, y)$ be any function of class $c^{k}$ on $R+\Gamma$ assuming the values of $g(x, y)$ on $\Gamma$. Define $b_{i}=D\left[\psi-g, f_{i}\right]$.

Then

$$
\left|\psi-g-\sum_{i=1}^{n} b_{i} f_{i}\right|=O\left(\sqrt{\frac{\sqrt{\theta(n)}}{n^{k-2}}} \log \frac{n}{(\log n)^{N}}\right),
$$

where

$$
D\left[\psi-g-\sum_{i=1}^{n} b_{i} f_{i}\right] \leq \begin{gathered}
\theta(n) \\
n^{k-2}
\end{gathered},
$$

with $\lim _{n \rightarrow \infty} \theta(n)=0, \theta$ depending on $\psi-g$, and where $N$ is any fixed constant $>0$. Moreover, if $k \geq 10$, then

$$
\left|\nabla \psi^{\prime}-\nabla\left(g+\sum_{i=1}^{n} b_{i} f_{i}\right)\right|=O\left(\left[\begin{array}{c}
\theta(n) \\
n^{k-10}
\end{array}\right]^{1 / 4}\right) .
$$

Finally, if $S$ is any closed domain in $R, k \geq 7$, then for points $P$ in $S$,

$$
\left|\nabla \psi-\Gamma\left(g+\sum_{i=1}^{n} b_{i} f_{i}\right)\right|=O\left(\left[\frac{\theta(n) \log n}{n^{k-6}}\right]^{1 / 4}\right) .
$$

Proof. Let $u_{n}=\psi-g-\sum_{i=1}^{n} b_{i} f_{i}$. Then $u_{n}$ is of the form $G \tau_{n}+H$ where the degree $m_{n}$ of $\tau_{n}$ is less than $\sqrt{ } 2 n-2$ and greater than $\sqrt{ } 2 n$ -2. By Theorem $1, D\left[u_{n}\right] \leq \theta(n) / n^{k-2}, k \geq 3$, where $\lim \theta(n)=0$ so that $D\left[u_{n}\right] \log m_{n} \leq A$ for some constant $A$ independent of $n$. By Lemma 7 ,

$$
\left|u_{n}\left(P_{0}\right)\right| \leq \sqrt{{ }_{\pi}^{2 \alpha}} D\left[u_{n}\right] \log ^{+} \frac{m_{n}}{\Delta_{n} D\left[u_{n}\right]}+B\left(\Delta_{n} D\left[u_{n}\right]\right)^{4} D\left[u_{n}\right]^{1 / 4}
$$

for any $\Delta_{n}>0$.

$$
\left|u_{n}\right| \leq \sqrt{\frac{2 \alpha}{\pi} D\left[u_{n}\right] \log ^{+} \frac{\sqrt{2 n}}{\Delta_{n} D\left[u_{n}\right]}}+B\left(\Delta_{n} D\left[u_{n}\right]\right)^{4} D\left[u_{n}\right]^{1 / 4} .
$$


There is a constant $E$, depending on $N$, such that $1 / n<E /(\log n)^{N} e$, $n \geq 3$. Then

$$
D\left[u_{n}\right] \leq \frac{\theta(n)}{n^{k-2}} \leq \frac{\theta(n) E^{k-2}}{e^{k-2}(\log n)^{N(k-2)}}=\frac{\sqrt{ } 2 n}{e^{k-2} \Delta_{n}} \leq \frac{\sqrt{ } 2 n}{e \Delta_{n}}
$$

if

$$
\Delta_{n}=\frac{\sqrt{ } 2 n(\log n)^{N(k-2)}}{\theta(n) E^{k-2}} .
$$

The function $x \log (\sqrt{2 n} / \Delta x)$ is monotone increasing for $0 \leq x \leq \sqrt{2 n} / e \Delta$ so that we may replace $D\left[u_{n}\right]$ by $\theta(n) / n^{k-2}$ to obtain

$$
\begin{aligned}
\left|u_{n}\right| \leq & \sqrt{\frac{2 \alpha}{\pi} n^{k-2}} \log ^{+} \frac{\theta(n) E^{k-2}}{(\log n)^{N(k-2)} \theta(n) / n^{k-2}} \\
& +B\left(\frac{\sqrt{2 n}(\log n)^{N(k-2)} \theta(n)}{\theta(n) E^{k-2}}\right)^{4}\left(\frac{\theta(n)}{n^{k-2}}\right)^{1 / 4} \\
= & O\left(\sqrt{\frac{V \theta(n)}{n^{k-2}} \log \frac{n}{(\log n)^{N}}}\right) .
\end{aligned}
$$

In the proof of Lemma 7 , we saw that $L<c_{6} m^{4}$ and $\sigma<c_{2} L M^{4}<$ $c_{2} c_{6} m^{8}$. Hence by equation (3) of Lemma 7,

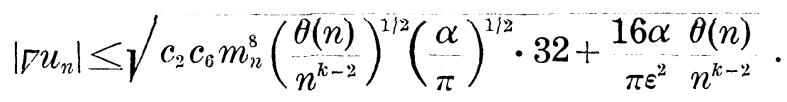

Since $m_{n}<\sqrt{2}$, we obtain the statement of the theorem regarding uniform convergence in $\bar{R}$ of $\left|\nabla u_{n}\right|$ for $k \geq 10$.

Next, let $S$ be any closed domain in $R$. We may suppose the boundary $I^{\prime \prime}$ of $S$ is sufficiently smooth so that a circle of radius $\varepsilon$ may be rolled around $\Gamma^{\prime}$ while lying in $S$. Let $L_{n}{ }^{\prime}=\sup _{S}\left|\tau_{n}\right|$ and $P_{0}^{(n)}$ be the point in $S$ where $L_{n}{ }^{\prime}=\mid \tau_{n}\left(P_{0}^{(n)} \mid\right.$. As in the proof of Lemma 7 ,

$$
\left|\nabla u_{n}\left(P_{0}\right)\right| \leq \sqrt{\left(\bar{\sigma}_{n}^{2} D\left[u_{n}\right]\right)^{1 / 2}\left(\begin{array}{c}
\alpha \\
\pi
\end{array}\right)^{1 / 2} \cdot 32+\frac{16 \alpha D\left[u_{n}\right]}{\pi \varepsilon^{2}}} \quad \text { for } P_{\Downarrow} \in S
$$

where

$$
\bar{\sigma}_{n}=G_{1} \underset{\varepsilon^{2}}{2 L_{n}^{\prime} m_{n}^{4}}+\underset{\varepsilon}{L_{n}^{\prime} m_{n}^{2}} G_{2}+L_{n}^{\prime} 2 G_{0}+\frac{G_{2} L_{n}^{\prime} m_{n}^{2}}{\varepsilon}+2 H_{0}
$$

Using $G \tau_{n}$ as the function $u$ of Lemma 8 defined over $\bar{R}$ and remembering that $G \tau_{n}=-\sum_{i=1}^{n} b_{i} f_{i}$, we obtain 


$$
\left|G\left(P_{0}^{(n)}\right) L_{n}^{\prime}\right| \leq \sqrt{D\left[\sum_{i=1}^{n} b_{i} f_{i}\right] \log m_{n} \mid c_{12}} .
$$

In $S,|G(P)| \geq c_{13}>0$. Also

$$
D\left[\sum_{i=1}^{n} b_{i} f_{i}\right]=\sum_{i=1}^{n} b_{i}^{2} \leq \sum_{i=1}^{\infty} b_{i}^{2}=D[\psi-g] .
$$

Therefore $L_{n}^{\prime}<c_{11} V \log n, \bar{\sigma}_{n}<c_{15} n^{2} \bigvee \log n$, and

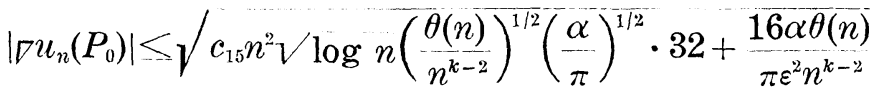

$$
\begin{aligned}
& =O\left(\left[\frac{\theta(n) \log n}{n^{k-6}}\right]^{1 / 4}\right) \text {. }
\end{aligned}
$$

Theorem 4. Let $R, \Gamma, G, f_{i}$ be defined as in Theorem 3. Then there is a constant $c_{17}$ such that whenever $P_{0} \in \bar{R}$, then

$$
\sum_{K=1}^{n} f_{K}^{2}\left(P_{0}\right) \leq c_{17} \log n
$$

The theorem is true if $\grave{P}_{0}$ is a point where $f_{1}, \cdots, f_{n}$ all vanish, in particular on $\Gamma$. Let $P_{0}$ be a point in $R$ where not all $f_{K}, K=1, \cdots$, $n$ vanish. Consider the problem of minimizing $D[u]$, where $u$ is of the form $u=\sum_{K=1}^{n} c_{K} f_{K}$, under the condition $u\left(P_{0}\right)=T \neq 0$. Now

$$
D[u]=D\left[\sum_{K=1}^{n} c_{K} f_{K}\right]=\sum_{K=1}^{n} c_{K}^{2},
$$

so that we must minimize $\sum_{K=1}^{n} c_{K}^{2}$ under the condition $\sum_{K=1}^{n} c_{K} f_{K}\left(P_{0}\right)=T$. By Lagrange multipliers we find a necessary condition for a minimum to be

$$
c_{K}=\bar{c}_{K}=\frac{T f_{K}\left(P_{0}\right)}{\sum_{j=1}^{n} f_{j}^{2}\left(P_{0}\right)},
$$

and the function $\bar{u}=\sum_{K=1}^{n} \bar{c}_{K} f_{K}$ satisfies

$$
D[\bar{u}]=T^{2} / \sum_{K=1}^{n} f_{K}^{2}\left(P_{0}\right) .
$$

This is actually a minimum value, for, if $u=\sum_{K=1}^{n} c_{K} f_{K}$, then

$$
T^{2}=\left[\sum_{K=1}^{n} c_{K} f_{K}\left(P_{0}\right)\right]^{2} \leq \sum_{K=1}^{n} c_{K}^{2} \sum_{K=1}^{n} f_{K}^{2}\left(P_{0}\right)
$$


so

$$
\frac{T^{2}}{\sum_{K=1}^{n} f_{K}^{2}\left(P_{0}\right)} \leq \sum_{K=1}^{n} c_{K}^{2}=D[u]
$$

Now $\bar{u}$ is of the form $G \tau_{n}$ where $\tau_{n}$ has the degree of $f_{n}$ and this degree is less than $\sqrt{2 n-2}$.

By Lemma 8, we have $D[\bar{u}] \geq c_{12} T^{2} / \log \sqrt{ } 2 n-2$.

Hence

$$
\begin{gathered}
T^{2}=D[\bar{u}] \geq \frac{c_{12} T^{2}}{\log \sqrt{2 n-2}}, \\
\sum_{K=1}^{n} f_{K}^{2}\left(P_{0}\right) \\
\sum_{K=1}^{n} f_{K}^{2}\left(P_{0}\right) \leq{ }_{c_{12}}^{1} \log \sqrt{2 n-2} .
\end{gathered}
$$

4. An Associated Problem. As in the previous sections, let $R$ be a bounded region whose boundary $\Gamma$ consists of a finite number of simply closed regular arcs of class $c^{k}, k \geq 3 ; G(x, y)$ be a function of class $c^{k}$ on $R+\Gamma$, vanishing on $\Gamma$, positive in $R$, with $\partial G / \partial \nu \geq \delta>0$ on $\Gamma ; g(x, y)$ be any function of class $c^{k}$ on $R+\Gamma$; a variation be a function of class $c^{k}$ on $R+\Gamma$ vanishing on $I$.

Let

$$
D^{p}[\xi]=\iint_{R}\left(a \xi_{x}^{2}+b \xi_{y}^{2}+c \xi^{2}\right)^{p} d x d y
$$

where $a>0, b>0, c \geq 0$ on $R+\Gamma ; a, b$, and $c$ are bounded and integrable on $\bar{R} ; p$ is a real number greater than or equal to 1 .

Assuming the existence of a function $\psi_{0}$, yielding minimum value to $D^{p}[\psi]$ in the set of admissible functions of class $c^{k}$ on $R+\Gamma$, which take the value of $g$ on $I^{r}$, can we obtain $\psi_{0}$ by the Rayleigh Ritz method? This question is answered in the affirmative and an estimate is obtained for the rate of convergence.

Let $\|\xi\|=\left(D^{p}[\xi]\right)^{1 / 2 p}$, for $\xi$ in the set of functions of class $c^{k}$ on $R+\Gamma$. This functional has the properties $\|\xi\| \geq 0,\|a \xi\|=|a|\|\xi\|$ for real $a$, $\|\xi+\eta\| \leq\|\xi\|+\|\eta\|$.

The functional $\|\xi\|$ is a true norm in the linear space $V$ of variations. Let $H$ be the Banach space formed by completing $V$ with respect to this norm. As in the proof of Theorem 1, we see that the set of functions $G \tau$, where $\tau$ is a polynomial in $x$ and $y$, is dense in $H$. Moreover, if $\psi$ is admissible, there exists a sequence of polynomials $Q_{j}$ of degree at most $j$ such that 


$$
\| \psi-g-\left(\dot{r} Q_{j} \|=D^{\eta}\left[\psi^{\prime}-g-G Q_{j}\right]^{1 / 2 n}=O()\left(\frac{A(j)}{j^{k-2}}\right),\right.
$$

where $\theta$ depends on $\psi-g$ and $\lim _{j \rightarrow \infty} \theta(j)=0$.

There exists inf $D^{p}\left[\psi^{\prime}\right] \geq 0$ for admissible $\psi^{\prime}$. Let $\tau_{j}$ be a polynominal of degree at most $j$ which makes $D^{p}\left[g+G \tau_{j}\right] \leq D^{p}\left[g+G Q_{j}\right]$ for all polynomials $Q_{j}$ of degree at most $j$.

That such a polynomial $\tau_{j}$ exists can be seen as follows. The class of all functions $G Q_{j}$ where $Q_{j}$ is a polynominal of degree at most $j$ is also the linear manifold determined by $f_{i}=G T_{i}$, the orthonormal sequence of Theorem 1, whose polynomial factor $T_{i}$ is of degree at most $j$. As stated in the introduction, $1 \leq i \leq j\left(\begin{array}{c}j+1 \\ 2\end{array}\right)+j+1=\sigma$ so that we may write $G Q_{j}=\sum_{i=1}^{\sigma} c_{i} f_{i}$. Now let $Q_{j}^{\prime}$ be any fixed $Q_{j}$. We may restrict ourselves to those $Q_{j}$ such that $D^{p}\left[g+G Q_{j}\right] \leq D^{p}\left[G+G Q_{j}^{\prime}\right]$. For such $Q_{\text {, }}$ we have

$$
\|g\|+\left\|g+G Q_{j}^{\prime}\right\| \geq\|g\|+\left\|g+G Q_{j}\right\| \geq\left\|G Q_{j}\right\| .
$$

Since $D[\xi] \leq D^{p}[\xi]^{1 / p}|R|^{1 / q}$ where $(1 / p)+(1 / q)=1,|R|=$ area of $R$, we find that

$$
|R|^{1 / 4}\left[\|g\|+\left\|g+G Q_{j}^{\prime}\right\|\right]^{2} \geq D\left[G Q_{j}\right]=D\left[\sum_{i=1}^{\sigma} c_{i} f_{i}\right]=\sum_{i=1}^{\sigma} c_{i}^{2} .
$$

Thus the permissible $c_{i}$ lie in a bounded closed set $S$ in $\sigma$-dimensional space. Since

$$
D^{p}\left[g+G Q_{j}\right]=D^{p}\left[g+\sum_{i=1}^{\sigma} c_{i} f_{i}\right]
$$

is a continuous function of $c_{i}$ in $S$, it attains its minimum in $S$.

Since $D^{p}\left[g+G \tau_{j}\right]$ is a decreasing function of $j$, we have

$$
\lim _{j \rightarrow \infty}\|g+G \tau,\| \leq \liminf _{j \rightarrow \infty}\left\|g+G Q_{j}\right\| .
$$

Let $\psi$ be admissible and choose $Q_{j}$ so that $\lim _{j \rightarrow \infty} D^{p}\left[\psi-g-G Q_{j}\right]=0$. Then $\left\|g+G Q_{j}\right\| \leq\|\psi\|+\left\|\psi-g-G Q_{j}\right\|$ implies that $\liminf \left\|g+G Q_{j}\right\| \leq\|\psi\|$. It follows that $\lim _{j \rightarrow \infty}\left\|g+G \tau_{j}\right\| \leq\|\psi\|$ for every admissible $\phi$ and thus $g+G \tau_{j}$ is a minimizing sequence.

If $c>0$ in a set of positive measure in $R$, the functional $\|\xi\|$ is a true norm in the linear space $\left(c^{k}\right)$ of functions of class $c^{k}$ on $R+\Gamma$. If $c=0$, a.e. in $R$, this is still true provided we identify functions differing by a constant. In either case we will complete the space $\left(c^{k}\right)$ to form a Banach space $B$. 
A set $S$ in a normed linear space is uniformly convex if there exists a continuous monotone increasing function $g(\varepsilon), 0 \leq \varepsilon<1$, with $\lim _{\varepsilon \rightarrow \infty} g(\varepsilon)=0$, such that whenever $\xi, \eta$ are in $S$ and $\|\xi\|=\|\eta\|=1,\|(\xi+\eta) / 2\|$ $\geq 1-\varepsilon$, then $\|\xi-\eta\| \leq g(\varepsilon)$.

We shall show $\left(c^{k}\right)$ is uniformly convex. It is easily verified that if $\alpha, \beta$ are $\geq 0$, and $p \geq 1$ then

$$
3 \alpha^{p}+\beta^{p} \leq 2|\alpha+\beta|^{p}+|\alpha-\beta|^{p} .
$$

Apply the inequality to the integrand below, where we assume $\phi$ and $\psi$ are in $\left(c^{k}\right),\|\phi\|=\|\psi\|=1,\|(\phi+\psi) / 2\| \geq 1-\varepsilon$.

$$
\begin{aligned}
& \iint 3\left[a\left(\begin{array}{c}
\phi+\psi \\
2
\end{array}\right)_{x}^{2}+b\left(\begin{array}{c}
\phi+\psi \\
2
\end{array}\right)_{y}^{2}+c\left(\begin{array}{c}
\phi+\psi \\
2
\end{array}\right)^{2}\right]^{p} \\
& \quad+\left[a\left(\begin{array}{c}
\phi-\psi \\
2
\end{array}\right)_{x}^{2}+b\left(\begin{array}{c}
\phi-\psi \\
2
\end{array}\right)_{y}^{2}+c\left(\begin{array}{c}
\phi-\psi \\
2
\end{array}\right)^{2}\right]^{p} d x d y \\
& \leq 2^{2} \iint\left[\left(a \phi_{x}^{2}+b \phi_{y}^{2}+c \phi^{2}\right)+\left(a \psi_{x}^{2}+b \psi_{y}^{2}+c \psi^{2}\right)\right]^{p} d x d y \\
& \quad+\iint\left|a \phi_{x} \psi_{x}+b_{y} \phi_{y} \psi_{y}+c \phi \psi\right|^{p} d x d y \\
& \leq \begin{array}{l}
2 \\
2^{p}
\end{array}\left\{\left(\iint\left(a \phi_{x}^{2}+b \phi_{y}^{2}+c \phi^{2}\right)^{p} d x d y\right)^{1 / p}+\left(\iint_{R}\left(a \psi_{x}^{2}+b \psi_{y}^{2}+\psi c^{2}\right)^{p} d x d y\right)^{1 / p}\right\}^{p} \\
& \quad+\iint\left(\sqrt{ } a \phi_{x}^{2}+b \phi_{y}^{2}+c \phi^{2} V a \psi_{x}^{2}+b \psi_{y}^{2}+c \psi^{2}\right)^{p} d x d y \\
& \leq 2+\sqrt{ } \iint\left(a \phi_{x}^{2}+b \phi_{y}^{2}+c \phi^{2}\right)^{p} d x d y / \iint\left(a \psi_{x}^{2}+b \psi_{y}^{2}+c \psi^{2}\right)^{p} d x d y=3
\end{aligned}
$$

Hence

$$
\iint\left(a\left(\begin{array}{c}
\phi-\psi \\
2
\end{array}\right)_{x}^{2}+b\left(\begin{array}{c}
\phi-\psi \\
2
\end{array}\right)_{y}^{2}+c\left(\begin{array}{c}
\phi-\psi \\
2
\end{array}\right)^{2}\right)^{p} d x d y \leq 3-3(1-\varepsilon)^{2 p}
$$

and

$$
\begin{aligned}
&\|\phi-\psi\|=\left[\iint\left(a\left(\phi-\psi^{\prime}\right)_{x}^{2}+b(\phi-\psi)_{y}+c(\phi-\psi)^{2}\right)^{p} d x d y\right]^{1 / 2 p} \\
& \leq 2\left(3\left[1-(1-\varepsilon)^{2 p}\right]\right)^{1 / 2 p} \leq 2\left(3^{1 / 2 p}\right)(2 p \varepsilon)^{1 / 2 p}=g(\varepsilon)
\end{aligned}
$$

for $\varepsilon<1$, since the function $y=\left[1-(1-x)^{2 p}\right]-2 p x$ vanishes at 0 and is a decreasing function of $x$ for $0 \leq x<1$.

Lemma 9. Let $B$ be a Banach space, $Y$ a set in $B$ with the property that if $y_{1}, y_{2}$ are in $Y$, then so is $\left(y_{1}+y_{2}\right) / 2$. Let the linear manifold spanned by $Y$ be a uniformly convex set in $B$. Let 


$$
\rho=\inf _{y \in Y}\|y\|>0,
$$

let $y_{n}$ be a sequence in $Y$ with

$$
\lim _{n \rightarrow \infty}\left\|y_{n}\right\|=\rho, \rho_{n}=\left\|y_{n}\right\| \text {. }
$$

Then there exists a unique $x$ in $B$ such that $\|x\|=\rho$ and we have

$$
\left\|x-y_{n}\right\| \leq \rho g\left(\frac{\rho_{n}-\rho}{2 \rho}\right)+\rho_{n}-\rho,
$$

where $g(\varepsilon)$ is the function in the definition of uniform convexity. If $\rho=$ $\inf _{y \in Y}\|y\|=0$, and $\lim _{n \rightarrow \infty}\left\|y_{n}\right\|=\rho$, then there is a unique $x$ in $B$ such that $\|x\|=\rho$, and we have $\left\|x-y_{n}\right\|=\rho_{n}-\rho$.

Proof. Let $z_{n}=y_{n} / \rho_{n}$ so that $\left\|z_{n}\right\|=1$. Write

$$
\begin{aligned}
& \frac{z_{n}+z_{m}}{2}-\frac{1}{\rho}\left(\frac{y_{n}+y_{m}}{2}\right)=\frac{y_{n}}{2}\left(\frac{1}{\rho_{n}}-\frac{1}{\rho}\right)+\frac{y_{m}}{2}\left(\frac{1}{\rho_{m}}-\frac{1}{\rho}\right) . \\
& \left\|\frac{z_{n}+z_{m}}{2}\right\| \geq \frac{1}{\rho}\left\|\frac{y_{n}+y_{m}}{2}\right\|-\frac{\left\|y_{n}\right\|}{2}\left(\frac{1}{\rho}-\frac{1}{\rho_{n}}\right)-\frac{\left\|y_{m}\right\|}{2}\left(\frac{1}{\rho}-\frac{1}{\rho_{m}}\right) \\
& \geq \frac{1}{\rho} \cdot \rho-\frac{\rho_{n}}{2}\left(\frac{1}{\rho}-\frac{1}{\rho_{n}}\right)-\frac{\rho_{m}}{2}\left(\frac{1}{\rho}-\frac{1}{\rho_{m}}\right)=1-\frac{\left(\rho_{n}-\rho\right)+\left(\rho_{m}-\rho\right)}{2 \rho} .
\end{aligned}
$$

Hence

$$
\left\|z_{n}-z_{m}\right\| \leq g\left(\frac{\left(\rho_{n}-\rho\right)+\left(\rho_{m}-\rho\right)}{2 \rho}\right) \text { for } \frac{\rho_{n}-\rho+\rho_{m}-\rho}{2 \rho}<1
$$

Thus there exists $z=\lim _{n \rightarrow \infty} z_{n}$ in $B$. Let $x=\rho z=\lim _{n \rightarrow \infty} \rho z_{n}=\lim _{n \rightarrow \infty} \rho_{n} z_{n}=\lim _{n \rightarrow \infty} y_{n}$. Then $\|x\|=\lim _{n \rightarrow \infty}\left\|y_{n}\right\|=\rho$. Also $\left\|z_{n}-z\right\| \leq g\left(\left(\rho_{n}-\rho\right) / 2 \rho\right)$ implies

$$
\left\|x-y_{n}\right\|=\left\|\rho z-\rho_{n} z_{n}\right\| \leq\left\|\rho z-\rho z_{n}\right\|+\left\|\rho z_{n}-\rho_{n} z_{n}\right\| \leq \rho g\left(\frac{\rho_{n}-\rho}{2 \rho}\right)+\rho_{n}-\rho .
$$

To show $x$ is unique, suppose also $y_{n}^{\prime} \in Y, \lim _{n \rightarrow \infty}\left\|y_{n}^{\prime}\right\|=\rho, x^{\prime} \in B,\left\|x^{\prime}\right\|=\rho$, $x^{\prime}=\lim _{n \rightarrow \infty} y_{n}^{\prime}$. Then form the sequence $\left\{y_{n}^{\prime \prime}\right\}=y_{1}, y_{1}^{\prime}, y_{2}^{\prime}, y_{2}^{\prime}$, etc. of elements of $Y$ with $\lim _{n \rightarrow \infty} y_{n}^{\prime \prime}=\rho$. As above, $\forall x^{\prime \prime} \in B$ with $x^{\prime \prime}=\lim _{n \rightarrow \infty} y_{n}^{\prime \prime}=$ $\lim _{n \rightarrow \infty} y_{n}^{\prime}=\lim _{n \rightarrow \infty} y_{n}$. The last part of the lemma is obvious, since only $\|0\|=0$.

To apply the lemma, let $B$ be the completion of $\left(c^{k}\right), Y$ the set of admissible functions,

$$
y_{n}=g+G \tau_{n}, \quad \rho=\inf D^{p}[\psi]^{1 / 2 p},
$$

for admissible $\psi$. By the lemma, there is a unique $x$ such that $\|x\|_{=}=$ 
$\rho$. Assuming that $x=\psi^{\prime}$ is in $Y$, we can choose polynomials $Q$, of degree at most $j$ such that

$$
\left\|\psi_{0}-g-G Q_{j}\right\|=O\left(\frac{\theta(j)}{j^{k-2}}\right) .
$$

Then

$$
\begin{aligned}
\rho_{j}-\rho & =\left\|g+G \tau_{j}\right\|-\left\|\psi_{0}\right\| \leq\left\|g+G Q_{j}\right\|-\left\|\psi_{0}\right\| \\
& =\left\|g+G Q_{j}-\psi_{0}+\psi_{0}\right\|-\left\|\psi_{0}\right\| \leq\left\|\psi_{0}-g-G Q_{j}\right\|=O\left(\begin{array}{c}
\theta(j) \\
j^{k-2}
\end{array}\right) .
\end{aligned}
$$

By the lemma,

$$
\left\|\psi_{0}-g-G \tau_{j}\right\| \leq 2 \rho(6 p)^{1 / 2 p}\left(\frac{\rho_{j}-\rho}{2 \rho}\right)^{1 / 2 p}+\rho_{j}-\rho=O\left(\frac{\theta(j)}{j^{k-2}}\right)^{1 / 2 p},
$$

a better result, $O\left(\theta(j) / j^{k-2}\right)$, is obtained in the case $\rho=0$.

Since

$$
D[u]<\left(D^{p}[u]\right)^{1 / p}|R|^{1 / q},
$$

where $|R|$ is the area of $R$ and $(1 / p)+(1 / q)=1$, we find

$$
D\left[u_{j}\right] \leq\left(\frac{\theta(j)}{j^{k-2}}\right)^{1 / p},
$$

where $\lim _{j \rightarrow \infty} \theta(j)=0$, when we take $u_{j}=\psi_{0}-g-G \tau_{j}$. A proof similar to

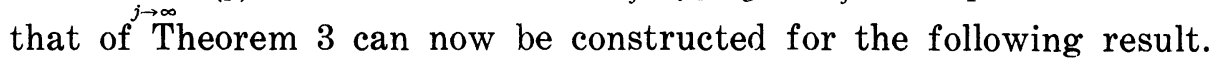

THEOREM 5. Let $R$ be a bounded region whose boundary $\Gamma$ consists of a finite number of simply closed regular arcs of class $c^{k}, k \geq 3$. Let $G(x, y)$ be a function of class $c^{k}$ on $R+\Gamma$, vanishing on $\Gamma$, positive in $R$, with $\partial G / \partial \nu \geq \delta>0$ on $\Gamma$. Let $a, b, c$ be bounded and integrable on $\bar{R}$, and $a>0, b>0, c \geq 0$ on $\bar{R}$. Let $g(x, y)$ be any function of class $c^{k}$ on $R+\Gamma$. Choose polynomials $\tau_{j}$ minimizing $D^{p}\left[g+G Q_{j}\right]$ in the set of all polynomials $Q_{j}$ of degree at most $j$. Then, if $\psi_{0}$ yields minimum value to $D^{p}[\psi]$ for $\psi$ in the set of functions of class $c^{k}$ on $R+\Gamma$ assuming the values of $g$ on $\Gamma$, we have

$$
\left|\psi_{0}-g-G \tau_{j}\right|=O\left(\sqrt{\left(\frac{\sqrt{\theta}(j)}{j^{k-2}}\right)^{1 / p}} \log \frac{j}{(\log j)^{N}}\right),
$$

where $N$ is any fixed positive constant, $\theta(j)$ depends on $\psi_{0}-g$ and $\lim _{j \rightarrow \infty} \theta(j)=0$. 
If $k \geq 16 p+2$, then

$$
\left|\nabla \psi^{\prime}{ }_{0}-\nabla\left(g+G \tau_{j}\right)\right|=O\left(\left[\begin{array}{c}
\theta(j) \\
j^{k-2-16 p}
\end{array}\right]^{1 / 4 p}\right) .
$$

\section{REFERENCES}

1. H. O. Herschfeld, "Continuation of differentiażle functions through the plane", Quart. J. Math. 7 (1930), 5.

2. L. Kantorovitch, "On the convergence of variational methods", Comptes Rendus (Doklady), Akademia Nauk. Vol. 30 (1941), 107-111.

3. O. D. Kellogg, “On bounded polynomials in several variables”, Math. Zeitschr. 27 (1928), 55-64.

4. N. Kryloff and N. Bogoliubov, "Application de la méthode de l'algorithme variationnel à la solution approchée des équations defférentielles aux dérivées partielles du typo elliptique", Akademia Nauk, Izvosta, (1930), 43-71.

5. E. L. Mickelson, "On the approximate representation of a function of two variables", Trans. Amer. Math. Soc. 33 (1931), 759-781.

6. H. Whitney, "Analytrc extensions of differentiable functions defined in closed sets", Trans. Amer. Math. Soc. 36 (1934), 63-89.

7. - "Functions differentiable on the boundaries of regions", Ann. Math. 35 (1934), 482-485.

UNIVERSITY OF MINNESOTA 



\section{PACIFIC JOURNAL OF MATHEMATICS}

\section{EDITORS}

\author{
H. L. Royden \\ Stanford University \\ Stanford, California \\ E. Hewite \\ University of Washington \\ Seattle 5 , Washington
}

\author{
R. P. Dilworth
}

California Institute of Technology Pasadena 4, California

\section{A. HorN*}

University of California

Los Angeles 24, California

\section{ASSOCIATE EDITORS}

\author{
H. BUSEMANN \\ HERBERT FEDERER \\ MARSHALL HALL
}

\author{
P. R. HALMOS \\ HEINZ HOPF \\ ALFRED HORN
}

\author{
R. D. JAMES \\ BORGE JESSEN \\ PAUL LÉVY
}

GEORGE PÓLYA

J. J. STOKER

KOSAKU YOSIDA

\section{SPONSORS}

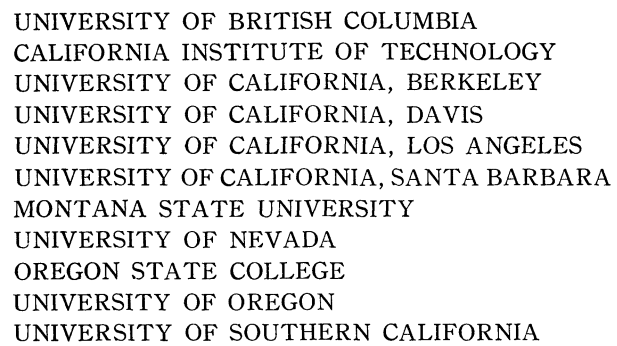

UNIVERSITY OF BRITISH COLUMBIA

CALIFORNIA INSTITUTE OF TECHNOLOGY

UNIVERSITY OF CALIFORNIA, BERKELEY

UNIVERSITY OF CALIFORNIA, DAVIS

UNIVERSITY OF CALIFORNIA, LOS ANGELES

UNIVERSITY OF CALIFORNIA, SANTA BARBARA

MONTANA STATE UNIVERSITY

UNIVERSITY OF NEVADA

OREGON STATE COLLEGE

UNIVERSITY OF OREGON

UNIVERSITY OF SOUTHERN CALIFORNIA

\author{
STANFORD RESEARCH INSTITUTE \\ STANFORD UNIVERSITY \\ UNIVERSITY OF UTAH \\ WASHINGTON STATE COLLEGE \\ UNIVERSITY OF WASHINGTON
}

AMERICAN MATHEMATICAL SOCIETY

HUGHES AIRCRAFT COMPANY SHELL DEVELOPMENT COMPANY

Mathematical papers intended for publication in the Pacific Journal of Mathematics should be typewritten (double spaced), and the author should keep a complete copy. Manuscripts may be sent to any of the editors. Manuscripts intended for the outgoing editors should be sent to their successors. All other communications to the editors should be addressed to the managing editor, Alfred Horn at the University of California Los Angeles 24, California.

50 reprints of each article are furnished free of charge; additional copies may be obtained at cost in multiples of 50 .

The Pacific Journal of Mathematics is published quarterly, in March, June, September, and December. The price per volume (4 numbers) is $\$ 12.00$; single issues, $\$ 3.50$; back numbers (Volumes $1,2,3)$ are available at $\$ 2.50$ per copy. Special price to individual faculty members of supporting institutions and to individual members of the American Mathematical Society: $\$ 4.00$ per volume; single issues, $\$ 1.25$.

Subscriptions, orders for back numbers, and changes of address should be sent to the publishers, University of California Press, Berkeley 4, California.

Printed at Kokusai Bunken Insatsusha (International Academic Printing Co., Ltd.) No. 10 1-chome Fujimi-cho, Chiyoda-ku, Tokyo, Japan.

* During the absence of E. G. Straus. 


\section{Pacific Journal of Mathematics}

\section{Vol. 5, No. $5 \quad$ BadMonth, 1955}

Henry A. Antosiewicz, A theorem on alternatives for pairs of matrice . . . . . 641

F. V. Atkinson, On second-order non-linear oscillation ............... 643

Frank Herbert Brownell, III, Fourier analysis and differentiation over real separable Hilbert spac .................................. 649

Richard Eliot Chamberlin, Remark on the averages of real function ...... 663

Philip J. Davis, On a problem in the theory of mechanical quadrature ... . . 669

Douglas Derry, On closed differentiable curves of order $n$ in $n$-spac ...... 675

Edwin E. Floyd, Boolean algebras with pathological order topologie ... . . 687

George E. Forsythe, Asymptotic lower bounds for the fundamental frequency of convex membrane ................................. 691

Israel Halperin, On the Darboux propert ................... 703

Theodore Edward Harris, On chains of infinite orde .............. 707

Peter K. Henrici, On certain series expansions involving Whittaker functions

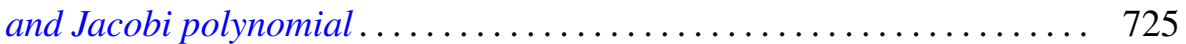

John G. Herriot, The solution of Cauchy's problem for a third-order linear hyperoblic differential equation by means of Riesz integral ......... 745

Jack Indritz, Applications of the Rayleigh Ritz method to variational

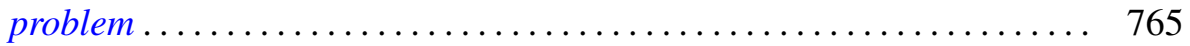

E. E. Jones, The flexure of a non-uniform bea ................. 799

Hukukane Nikaidô and Kazuo Isoda, Note on non-cooperative convex game.

Raymond Moos Redheffer and W. Wasow, On the convergence of asymptotic solutions of linear differential equation . . .

S. E. Warschawski, On a theorem of L. Lichtenstei ...........

Philip Wolfe, The strict determinateness of certain infinite game... 